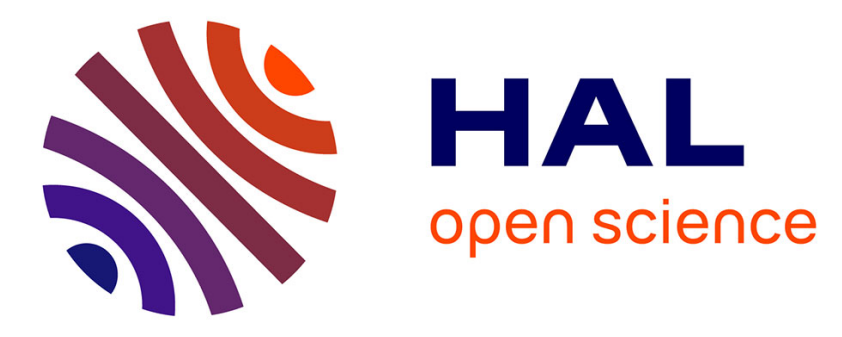

\title{
Impact of collisionality on turbulence in the edge of tokamak plasma using 3D global simulations
}

\author{
Raffaele Tatali, Eric Serre, Patrick Tamain, Davide Galassi, Philippe
}

Ghendrih, Federico Nespoli, Hugo Bufferand, Thomas Cartier-Michaud, Guido

Ciraolo

\section{To cite this version:}

Raffaele Tatali, Eric Serre, Patrick Tamain, Davide Galassi, Philippe Ghendrih, et al.. Impact of collisionality on turbulence in the edge of tokamak plasma using 3D global simulations. Nuclear Fusion, 2021, 10.1088/1741-4326/abe98b . hal-03182318

\section{HAL Id: hal-03182318 \\ https://hal.science/hal-03182318}

Submitted on 26 Mar 2021

HAL is a multi-disciplinary open access archive for the deposit and dissemination of scientific research documents, whether they are published or not. The documents may come from teaching and research institutions in France or abroad, or from public or private research centers.
L'archive ouverte pluridisciplinaire HAL, est destinée au dépôt et à la diffusion de documents scientifiques de niveau recherche, publiés ou non, émanant des établissements d'enseignement et de recherche français ou étrangers, des laboratoires publics ou privés. 


\title{
Impact of collisionality on turbulence in the edge of tokamak plasma using 3D global simulations
}

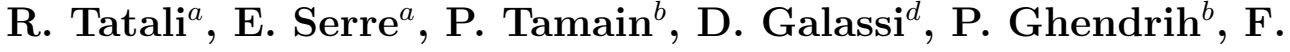

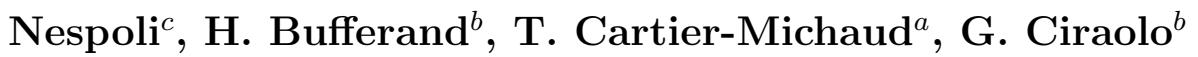 \\ ${ }^{a}$ Aix Marseille Univ., CNRS, Centrale Marseille, M2P2, France \\ ${ }^{b}$ IRFM, CEA-Cadarache, F-13108 Saint-Paul-lez-Durance, France \\ ${ }^{c}$ Princeton Plasma Physics Laboratory, 100 Stellaretor Road, Princeton, NJ 08540 \\ ${ }^{d}$ Ecole Polytechnique Fédérale de Lausanne (EPFL), Swiss Plasma Center (SPC), \\ 1015 Lausanne, Switzerland \\ E-mail: raffaele.tatali@gmail.com
}

\begin{abstract}
Collisionality is one of the key parameters in determining turbulent transport in the plasma edge, regulating phenomena such as "shoulder formation", separation of scale lengths in the scrape-off layer, turbulence damping and zonal flow dynamics. Understanding its role is therefore of primary importance for future reactors like ITER. Obtaining reliable predictions and a better characterization of plasma flow properties when varying collisionality remains, however, a critical challenge for the simulations. This paper focuses on the impact of varying collisionality in a nonisothermal three-dimensional fluid model of the plasma edge. A high field side limited configuration encompassing open and closed magnetic field lines with parameters typical of a medium-sized tokamak is considered. The present model can consistently account for the variations of collisionality and its impact on both the parallel resistivity $\eta_{\|}$and the ion and electron parallel thermal conductivities $\chi_{\| e, i}$. Details on mean flow and turbulence properties are given. Changing collisionality leads to significant changes in the flow properties both on the mean and fluctuating quantities. In particular, lowering collisionality decreases the size of coherent structures, the fluctuation levels of turbulence, and steepens the density and temperature equilibrium profiles around the separatrix leading to a global reduction of the turbulent transport. The scrape-off layer (SOL) width is observed to increase with collisionality, eventually resulting in the disappearance of the scale lengths separation between near and far SOL, consistently with previous experimental observations. At low collisionality, where the presence of narrow feature is well-established, a contribution of heat conduction increases up to compete with heat convection.
\end{abstract}

\section{Introduction}

A key issue for the success of future tokamaks is predicting and controlling the distribution of particle and heat loads striking the plasma facing components. This relies on a deeper understanding of transport processes in the scrape-off layer (SOL) 
and ultimately determining its width. Generally, it is claimed that transport along and across the magnetic field lines sets the SOL width [1]. In low confinement plasma (Lmode), experimental and theoretical results have shown that turbulent transport is the main cause for the cross-field fluxes [2 5].

A common feature observed in the SOL of many tokamaks during L-mode discharge is the so-called "shoulder formation": a rise of density decay length when a certain density threshold is overcome [6]. Generally, this is due to an enhancement of turbulence level that leads to the broadening of equilibrium profiles, eventually increasing the particle flux on the first wall and accelerating its deterioration. It has been suggested that this phenomenon is related to an increased collisionality [7, 8]. The collisionality is a measure of the importance of collisional processes in the plasma dynamics. It is usually defined as an adimensional quantity $\nu_{\star}$ using the electron-ion collision time $\tau_{e i}$ that depends on both density and temperature. Recent experiments have shown that the shoulder formation coincides both with the transition of filamentary structures into a new dynamic regime [9-11] and with the onset of the divertor detachment [12]. However, the role played by detachment is less clear, as pointed out in some experimental studies [13, 14]. Understanding the origin of the shoulder is a critical issue for future reactors, as ITER, which are expected to operate at high collisionality regimes, and with a partially detached divertor. If the shoulder is caused by a local increment of collisionality due to the detachment, it will strongly impact the operational space of ITER. Meanwhile, if it is the result of an overall collisionality's increment owing to density and temperature conditions in the plasma, it will likewise be suppressed due to the higher temperature available in bigger size machines [15, 16].

Besides, theoretical and numerical studies on the instability mechanisms in the edge region [17 19] have shown that collisionality is one of the control parameters which can change the instability dominating the plasma dynamic. In addition, the reduction of turbulent transport at low collisionality regimes is often invoked as a possible explanation for the L-H transition [20, 21].

Finally, it has been recognized that zonal flows should have a major role in the dynamics of turbulence and radial transport [22,24] and that collisionality can impact their dynamics [25, 26]. When plasma turbulence self-organizes through multi-scale interactions, zonal flows (toroidal and poloidal symmetric perturbation of electrostatic potential with a finite radial extend ) and large scale transport events (avalanches, streamers) play a crucial role on flow properties. Moreover, recent analyses in fluid turbulence [27, 28] have suggested that zonal flows could impact turbulence properties.

For all these reasons, the study of the impact of changing collisionality on edge turbulence properties is a growing interest topic.

In this work, we focus on the plasma edge region, which encompasses the magnetic open field lines of the Scrape-Off Layer (SOL), the plasma boundary layer in direct contact with the wall, and the outer part of the magnetic closed field lines region on both sides of the so-called separatrix.

Despite the rapid growth of computer speed and significant improvements in 
computer technology, (gyro)-kinetic simulations remain extremely costly from the computational point of view. It is particularly true in the near-wall region where particle recirculation requires addressing the electron and ion dynamics on the same footing and in a magnetic topology that is much more complex than in the core. Consequently, fluid approach based on drift-reduced Braginskii equations 29] remains standard one near the wall where the temperature is lower and the collisional mean free path significantly smaller than in the core. Various state-of-the-art 3D codes exist in the community to investigate edge plasma turbulence in various configurations (see as examples GRILLIX [30], GBS [31], BOUT $++[32]$ ). Here, simulations have been performed with the code TOKAM3X [33]. This code has been largely used in former studies, mostly in its isothermal version, assuming a single value of collisionality and examining the effect of turbulence on complex geometries and/or neutral physics [34 39].

The paper is organized as follows. The non-isothermal TOKAM3X model is briefly introduced in Sec. 2 together with the numerical setup. Sec. 3 defines the collisionality and discusses the related parameters in the fluid framework. In Sec. 4, flow properties are investigated in details in a simulation of reference performed at a given collisionality value. Sec. 5 analyses how these proprieties are modified when varying the collisionality. Finally, some concluding remarks are given in Sec. 6.

\section{Setup of TOKAM3X simulations}

\subsection{The physical and numerical model}

A circular limited geometry with the limiter placed on the high field side has been considered. The magnetic equilibrium is assumed to be fixed and axisymmetric. The computational domain encompasses closed and open magnetic field lines, which will be referred to as the core-edge region and the scrape-off layer (SOL), respectively. All magnetic flux surfaces are considered to be circular and centered on the magnetic axis.

TOKAM3X is a 3D turbulence code based on two-fluid, electrostatic, drift-reduced Braginskii equations in the plasma edge of tokamak [33]. By adopting a flux-driven approach, the code solves in versatile geometry all flow scales simultaneously without assuming any separation between mean profiles and fluctuations. A non-isothermal model is reckoned in the present work. The fluid equations are made dimensionless using reference scales given in Tab. 1. They correspond to plasma conditions typical of the COMPASS tokamak. Time and lengths scales are normalized to the reference ion Larmor radius $\rho_{L}^{0}=\sqrt{m_{i} T_{0}} / e B_{0}$ and the ion cyclotron frequency $1 / \Omega_{i}^{0}=m_{i} / e B_{0}$ with, $m_{i}$ the ion mass, $B_{0}$ the reference magnetic field, and $T_{0}$ the reference temperature that also defines the dimensionless electronic and ionic temperature $T_{e}$ and $T_{i}$. The dimensionless density $N$ and electrostatic potential $\Phi$ are normalized to the reference value $n_{0}$ and $T_{0} / e$, respectively, and velocities are normalized to the reference ion acoustic velocity $c_{s}^{0}=\sqrt{T_{0} / m_{i}}$. All the other normalization conventions are derived from the previous ones. Hereinafter all variables will be dimensionless, unless specified 
otherwise.

The dimensionless set of equations and all the related quantities are given in Appendix A. They couple the time evolution of six variables: the density $N$, the ion parallel momentum $\Gamma_{\| i}\left(=N u_{\| i}\right)$, the electron and ion energy $E_{e}=1.5 P_{e}$ and $E_{i}=1.5 P_{i}+$ $0.5 \Gamma_{\| i}^{2} / N$, respectively, the parallel current $J_{\|}$and the electrostatic potential in the form of vorticity $W . P_{e}=N T_{e}$ and $P_{i}=N T_{i}$ are the electron and ion pressure.

\begin{tabular}{|lc|cc|cc|}
\hline$\rho_{L}^{0}[\mathrm{~m}]$ & $6.88 \cdot 10^{-4}$ & $\Omega_{i}^{0}\left[s^{-1}\right]$ & $5.5 \cdot 10^{7}$ & $B_{0}[T]$ & 1.15 \\
\hline$n_{0}\left[\mathrm{~m}^{-3}\right]$ & $5 \cdot 10^{18}$ & $R_{0}[\mathrm{~m}]$ & 0.56 & $\eta_{0}[\Omega m]$ & 1.43 \\
\hline$T_{0}[\mathrm{eV}]$ & 30 & $m_{i} / m_{e}$ & 2000 & $\chi_{0}\left[\Omega m e V^{-1}\right]$ & 0.047 \\
\hline
\end{tabular}

Table 1: Reference scales in SI typical of the COMPASS tokamak characteristics. $\rho_{L}^{0}$ is the Larmor radius, $n_{0}$ and $T_{0}$ are the reference density and temperature at separatrix, $\Omega_{i}^{0}$ is the ion cyclotron frequency, $\eta_{0}=B_{0} / e n_{0}$ is the parallel resistivity of reference, $\chi_{0}=B_{0} / e n_{0} T_{0}$ is the conductivity of reference and $m_{i, e}$ is the mass of ion and electron.

The direction of magnetic field is such that ion $\nabla B$ drift (defined in Appendix A ) is positive at the bottom of the machine. TOKAM3X has been verified with the Method of Manufactured Solutions (MMS) and more recently by using the data mining iPoPe method [40] for the isothermal part of the equations. The numerical scheme is based on an advanced second-order accurate finite-differences/finite-volumes method. It is associated with a second-order WENO reconstruction to solve the advection terms to handle shocks. This approach is thought to be highly conservative.

\subsection{Boundary conditions}

In the radial direction, homogeneous Neumann boundary conditions are imposed at the inner and outer boundaries for all variables.

In the open field lines region, Bohm-Chodura conditions are enforced in the parallel direction. They model the physics of the sheath located next to the limiter wall and in which quasi-neutrality is no longer valid [1]. These conditions impose a sonic velocity $c_{s}\left(=\sqrt{T_{e}+T_{i}}\right)$ and a parallel current $J_{\|}$on the two opposite sides of the limiter target plates. In a dimensionless form, they write as:

$$
\begin{aligned}
& \left|\Gamma_{\| i}\right| \geq N \sqrt{T_{e}+T_{i}} \\
& J_{\|}=j_{\text {sat }}\left[1-e^{\left(\Lambda-\frac{\Phi}{T_{e}}\right)}\right]
\end{aligned}
$$

where $\Lambda$ is the sheath floating potential (typically $\Lambda \simeq-3$ ) and $j_{\text {sat }}$ is the ion saturation current equal to $\pm N c_{s}= \pm N \sqrt{T_{e}+T_{i}}$. These conditions can be expressed likewise by introducing the Mach number $M=u_{\| i} / c_{s}$ and imposing that $|M| \geq 1$. Then, by combining the $J_{\|}$in Eq. 1 with the generalized Ohm's Law (Eq. A.6), it is possible to get the Robin boundary condition on the parallel gradient of the electric 
potential as:

$$
\nabla_{\|} \Phi=T_{e} \nabla_{\|} \log (N)+1.71 \nabla_{\|} T_{e} \pm \eta_{\|} \sqrt{T_{e}+T_{i}}\left[1-e^{\left(\Lambda-\frac{\Phi}{T_{e}}\right)}\right]
$$

It is further required that the second derivative of the density is fixed to 0 and $\partial_{\theta} W=0$ to suppress the diffusive current reaching the target. Regarding the electron and ion temperatures, assuming that the total heat exhaust in the parallel direction by the plasma is equal to $\gamma_{e / i} N T_{e / i} M$, being $\gamma_{i}=2.5$ and $\gamma_{e}=4.5$ the sheath heat transmission coefficients for ion and electron [1], the boundary conditions write as:

$$
\begin{aligned}
& \frac{5}{2} N M T_{e}-\chi_{\|, e} \nabla_{\|} T_{e}=\gamma_{e} N M T_{e} \\
& \frac{5}{2} N M T_{i}-\chi_{\|, i} \nabla_{\|} T_{i}=\gamma_{i} N M T_{i}
\end{aligned}
$$

\subsection{Numerical setup}

The minor and major radii of the tokamak are $a=334 \rho_{L}(0.23 m)$ and $R_{0}=$ $814 \rho_{L}(0.56 \mathrm{~m})$ respectively, and the aspect ratio is 2.4 , corresponding to COMPASSlike geometrical parameters. The safety factor varies radially, with $q_{95}^{s e p} \simeq 4$ at the last closed flux surface.

For numerical stability at the border, a very thin buffer region (over four radial points for the grids considered here) is introduced at each radial boundary in which an artificially very high diffusion is imposed to homogenize the plasma field over the first flux surfaces (see in [41]). These two buffer regions will be excluded from our analysis unless specified.

The total particle and energy fluxes are supposed to come from the plasma core. The sources are assumed to have a Gaussian shape with half-width $a / 16$ and to extend in the radial direction over the buffer region. The amplitudes of particle and energy sources are tuned in order to obtain roughly the reference density and temperature at the separatrix in the reference simulation of our scan (i.e., $N \simeq 1$ and $T \simeq 1$ in dimensionless units). The corresponding integrated sources are equal to $7.5 \cdot 10^{3}$ and $1.5 \cdot 10^{4}$ in dimensionless units, respectively, for $S_{N}$ and $S_{E e / i}$ that correspond to $6.7 \cdot 10^{20} \mathrm{~s}^{-1}$ and $7.5 \mathrm{~kW}$ (on each species) in SI units with the reference parameters of Table 1. The latter number might seem particularly low compared with experiments, but it can be explained by the fact that simulations are run in half-torus only and without including any energy loss due to ionization or radiating impurities. The sources are then kept constant in dimensionless units for all the simulations presented in this paper. A sketch of the buffer region and sources is shown in Figure 1 .

Finally, the perpendicular diffusion coefficients are chosen equal to $D_{N / \Gamma / T}=$ $5 \cdot 10^{-3} \rho_{L}^{2} \omega_{i}$ (i.e. $0.14 m^{2} s^{-1}$ ). They are kept constant in space and time throughout all discussed simulations. These effective diffusion terms can be interpreted as sub-grid transport terms and are effectively used to damp fluctuations at the discretization grid scale for numerical stability reasons. One can interpret them as accounting for both 
the collisional transport and the effect of the turbulent small scales (smaller than the grid spacing) in the cross-field direction. However, no dependency with local plasma conditions (see [42] for an example of such approach) is taken into account. The value used in this work results thus from a trade-off between the grid size requirement (directly related to the computational cost) and the relevant physics.

The grid resolution in the radial $\Psi$, poloidal, $\theta$ and toroidal direction $\varphi$, is equal to $N_{\Psi} \times N_{\theta} \times N_{\varphi}=64 \times 512 \times 64$ over the half-torus, implying $\pi$-periodicity for the solution in the toroidal direction. The output sampling frequency is $1 / 100 \omega_{c}$. The quasi-steadystate is assumed to be reached when the integrated density, electron and ion energies over a flux surface are almost constant over time, or likewise, the oscillations amplitude of time derivatives of the same quantities are less than $5 \%$. Hereinafter, we will use the radial coordinate $r_{u}=\Psi-\Psi_{\text {sep }}$, where $\Psi_{\text {sep }}$ is the separatrix location, thereby $r_{u}$ is 0 at the separatrix. By using $r_{u}$, the radial computational domain extends over the range $\left[-64 \rho_{L}, 64 \rho_{L}\right]$ from which the buffer regions are subtracted leaving an effective radial computational domain extending in the range $\left[-58 \rho_{L}, 58 \rho_{L}\right]$.

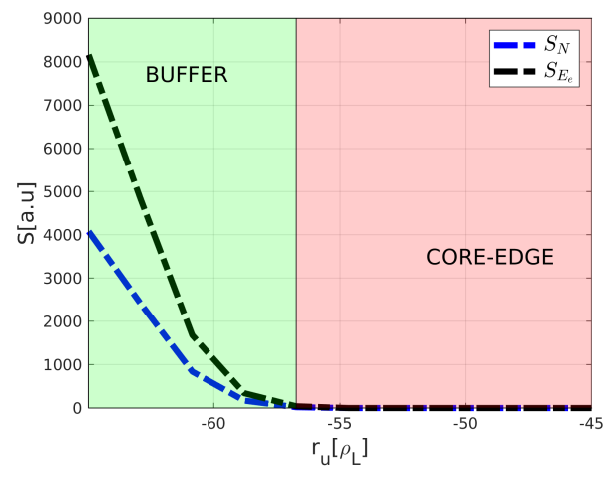

Figure 1: Radial profiles of the particle and energy sources integrated over the flux surface taken at $-64 \rho_{L}<r_{u}<-45 \rho_{L} \cdot r_{u}=\Psi-\Psi_{\text {sep }}$ is the distance from the separatrix. The green area $-64 \rho_{L}<r_{u}<-58 \rho_{L}$ is the buffer region at the inner boundary. It will no longer appear in the next figures unless specified otherwise. The same goes for the buffer region at the outer boundary $58 \rho_{L}<r_{u}<64 \rho_{L}$ (not displayed). The red area represents the beginning of the core-edge region.

\section{Collisionality}

The collisionality is the control parameter of the present study with $\nu_{\star}$ defined as:

$$
\nu_{\star}=\nu_{e i}^{0} \tau_{0 \|}^{i}
$$

where $\nu_{e i}^{0}$ is the inverse of the electron-ion collision time. It is a constant value determined once the reference temperature $T_{0}$ and density $n_{0}$ are fixed. $\tau_{0 \|}^{i}$ is chosen to be equal to the parallel transit time of ions in order to match the usual definition of the dimensionless resistivity [10]: $\tau_{0 \|}^{i}=L_{\|}^{\text {sep }} / c_{s}^{0}$, where $L_{\|}^{\text {sep }}=2 \pi q_{95}^{\text {sep }} R_{0}$ is the parallel 
connection length evaluated at the last closed flux surface $\left(q_{95}^{\text {sep }} \simeq 4\right.$ in the considered cases). Note that this is actually not the normalization of the collisionality $\nu_{\star}^{T K 3 X}$ used in the TOKAM3X model (see Appendix A.1) which does not depend on geometrical parameters. Practically speaking, since the geometry is held fixed throughout this study, the two definitions only differ by a multiplicative constant.

Based on the COMPASS-like parameters defined in the section above (Tab.1), we define a reference collisionality $\nu_{\star, \text { ref }}$ using $\tau_{e i}^{r e f} \simeq 25 \mu \mathrm{s}$.

$$
\begin{aligned}
\nu_{\star}, r e f & =\nu_{e i}^{r e f} \tau_{0 \|}^{i} \simeq 1500 \\
\nu_{\star} & =C \nu_{\star, r e f}
\end{aligned}
$$

In this study, we scan the collisionality $\nu_{\star}$ by varying the constant $\mathrm{C}$ in the range $[0.3-1000]$. In the fluid drift-reduced Braginskii model used here, collisionality determines both the parallel resistivity $\eta_{\|}$and the ion and electron thermal conductivities $\chi_{\| e, i}$ defined as:

$$
\begin{aligned}
& \eta_{\|}=0.51 \frac{m_{e}}{m_{i}} \frac{\rho^{\star}}{2 \pi q_{95}^{s e p} \mathcal{A}} \nu_{\star} T_{e}^{-\frac{3}{2}}=\eta_{\| 0} T_{e}^{-\frac{3}{2}} \\
& \chi_{\| e}=3.16 \frac{m_{i}}{m_{e}} \frac{2 \pi q_{95}^{s e p} \mathcal{A}}{\rho_{\star}} \frac{1}{\nu_{\star}} T_{e}^{\frac{5}{2}}=\chi_{\| 0 e} T_{e}^{\frac{5}{2}} \\
& \chi_{\| i}=3.9 \sqrt{\frac{2 m_{i}}{m_{e}}} \frac{2 \pi q_{95}^{s e p} \mathcal{A}}{\rho_{\star}} \frac{1}{\nu_{\star}} T_{i}^{\frac{5}{2}}=\chi_{\| 0 i} T_{i}^{\frac{5}{2}}
\end{aligned}
$$

$m_{e, i}$ are the electron and ion mass, $\mathcal{A}$ is the aspect ratio and $\rho^{\star}=\rho_{L} / a$. The presence of the geometrical parameters $\mathcal{A}$ and $q_{\text {sep }}$ in these expressions is due to the dependency in $L_{\|}^{s e p}$ of the definition of $\nu_{\star}$. A complete definition of the quantities in Eqs. (5) and (6) is given in Appendix A.1. Thus, the collisionality scan leads to a variation of the parallel resistivity $\eta_{\|}$and the thermal conductivities $\chi_{\| e / i}$ as well. In order to interpret the meaning of the $\nu_{\star}$ scan performed here with respect to experiments, let us note that scanning $\nu_{\star}$ while holding $\rho_{\star}$ constant for a given machine geometry is equivalent to scanning the reference density $n_{0}$. Since the driving fluxes (the amplitude of source terms) in SI units are proportional to $n_{0}$, this means that both the particle and energy sources in SI units are scanned accordingly. In other words, the reported dimensionless collisionality scan is representative of an experiment in which the particle fuelling and the heating power would be scanned together, leading to an increase of the density and a relatively constant temperature (put aside the impact of turbulent transport on the shape of profiles).

The choice of the COMPASS-like parameters has been made because we are able to simulate the actual size of the machine in terms of $\rho_{\star}$. Note however that the reference value $\nu_{\star, \text { ref }}$, has been increased by a factor of 3 compared to the collisionality that would be given by the reference parameters given in Tab. 1. This choice is the result of a compromise between computational cost (increasing with decreasing collisionality) and fidelity with respect to the experiment. It also allows comparisons with previously published TOKAM3X results [35]. This way, $0.3 \nu_{\star, \text { ref }}$ actually corresponds to $\tau_{\text {ei }}^{0}$, which 
is the experimental value measured in COMPASS. On the other side, the two simulations at 100 and $1000 \nu_{\star, \text { ref }}$ correspond to much higher resistivity and conductivity values than the ones met in experiments. Even so, they allow us, in our theoretical model, to stress flow properties in high collisionality regimes.

\begin{tabular}{|c|c|c|c|}
\hline$\nu_{*}$ & $\eta_{\| 0}$ & $\chi_{\| 0 e}$ & $\chi_{\| 0 i}$ \\
\hline \hline $1000 \nu_{\star, \text { ref }}$ & $10^{-2}$ & $1.61 \cdot 10^{2}$ & 4.64 \\
$100 \nu_{\star, \text { ref }}$ & $10^{-3}$ & $1.61 \cdot 10^{3}$ & $4.64 \cdot 10^{1}$ \\
$10 \nu_{\star, \text { ref }}$ & $10^{-4}$ & $1.61 \cdot 10^{4}$ & $4.64 \cdot 10^{2}$ \\
$\nu_{\star, \text { ref }}$ & $10^{-5}$ & $1.61 \cdot 10^{5}$ & $4.64 \cdot 10^{3}$ \\
$0.3 \nu_{\star, \text { ref }}$ & $3 \cdot 10^{-6}$ & $5.50 \cdot 10^{5}$ & $1.58 \cdot 10^{4}$ \\
$0.3 \nu_{\star, \text { ref }}$ & $3 \cdot 10^{-6}\left(\eta_{\|}(T)\right)$ & $5.50 \cdot 10^{5}$ & $1.58 \cdot 10^{4}$ \\
\hline
\end{tabular}

Table 2: Scan in dimensionless collisionality $\nu_{\star}$. Corresponding dimensionless values of the parallel resistivity $\eta_{\| 0}$ and the thermal conductivity $\chi_{\| 0 e i}$.

In all simulations $\eta_{\|}$is considered as constant and equal to $\eta_{\| 0}$, except for one case where the temperature-dependence in $T_{e}^{-3 / 2}$ has been taken into account (Eq.6. . On the contrary, the thermal conductivities $\chi_{\| e / i}$ always vary with temperature and the $\chi_{\| 0}$ for electron and ion are given in Tab. 2. Typical radial profiles of $\eta_{\|}\left(T_{e}\right)$ and $\chi_{\| e}\left(T_{e}\right)$ are illustrated on Fig. 2, They clearly show that they can behave very differently into the core edge region and the SOL. Profiles of $\chi_{\| i}\left(T_{i}\right)$ are not shown here because very similar to $\chi_{\| e}\left(T_{e}\right)$. Finally, we notice that the electron thermal conductivity is always larger than the ion thermal conductivity.
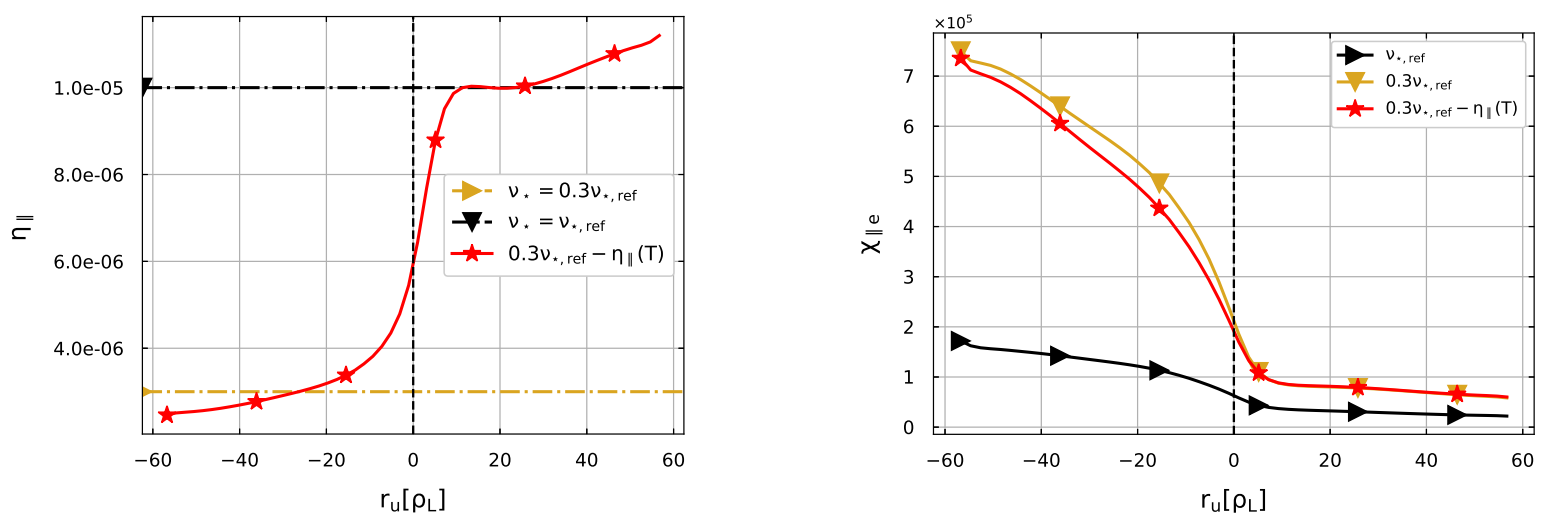

Figure 2: Numerical radial profiles of the parallel resistivity $\eta_{\|}$(left) and the electron thermal conductivity $\chi_{\| e}$ (right) averaged in time and $\varphi$ direction depending on the collisionality $\nu_{\star}$. The case $0.3 \nu_{\star}$, ref $-\eta_{\|}\left(T_{e}\right)$ corresponds to the case (Eq. 6) where the electron temperature dependence is taken into account. For the parallel conductivities the temperature dependence is always included. The dashed line corresponds to the radial location of the separatrix and $r_{u}$ is the distance from it.

Remark 1 - While changing the collisionality changes both the parallel resistivity $\eta_{\|}$and the thermal conductivities $\chi_{\| e / i}$, we may expect that the impact of changing the 
thermal conductivity is weaker. Indeed, on one side $\eta_{\|}$governs the electric potential into the vorticity equation and determines both $k_{\perp}^{2} \tilde{\Phi}$ and $\tilde{u}_{E \times B}$. On the other side, the thermal conductivity $\chi_{\| e / i}$ can be interpreted as a damping term in the energy equations acting on the parallel length scales only. It tends to reduce $k_{\|}$which is naturally small (fast dynamics in the parallel direction) and so its effect is expected to be small. This has been confirmed here by supplementary simulations (not shown in the paper) performed at a fixed parallel resistivity $\eta_{\|}=10^{-5}$ and a self-consistent variation of the $\chi_{\| e, i}$, for both $\nu_{\star}=0.3$ and $100 \nu_{\star}, r e f$. No significant change in the solutions has been observed on perpendicular transport and turbulent structures for these two values.

Remark 2 - In the SOL, the sheath at the limiter introduces an additional resistance $R_{\text {sheath }}$ that can be much higher than $R_{\|}\left(=\eta_{\|} L_{\|}\right)$. In the TOKAM3X model, an estimate of $R_{\text {sheath }}=T_{e} / j_{\text {sat }}$ together with among $R_{\|}$and $R_{\text {sheath }}$ is provided in Appendix B. Therefore, in this flow region, the electrostatic potential profile may be set through the Bohm boundary condition that could govern the whole dynamic.

\section{Reference case}

This simulation has been performed to serve as a reference to all other simulations of the paper. Both plasma equilibrium and turbulence properties of a limited non-isothermal plasma are detailed. The parallel resistivity $\eta_{\|}$is assumed to be constant here and equal to $\eta_{\| 0}=10^{-5}$. The parallel thermal conductivities depend on the temperatures with the values of $\chi_{0, e / i}$ provided in Tab.2.

\subsection{Edge and SOL turbulence}

Fig. 3 shows a typical 3D snapshot of the turbulent solution, characterized by density and temperature fluctuations. In agreement with former isothermal studies in a similar configuration [35], the reference solution shows that plasma turbulence is self-organized with the occurrence of coherent structures in the core-edge region (closed field lines) and in the SOL (open field lines). Those bursts of fluctuations, called filaments and known to be the main responsible for the total radial flux of particles in the SOL [43], expand along the magnetic field lines in the toroidal direction and propagate outwardly via ballistic mechanism. They exhibit different sizes in the poloidal plane, with little radial coherence in the edge of the core region, and with a larger coherence in both radial and poloidal directions into the SOL. This change across the separatrix has been already observed in many experiments on several machines [5] and corresponds to a rapid change of the turbulence statistics as already documented experimentally [44].

The radial evolution of the Probability Distribution Function (PDF), skewness and kurtosis at the LFS of the reference case is shown in Fig. 4.

For a general scalar $X$ the skewness and excess kurtosis are defined as: $\mathcal{S}=$ $\frac{\left\langle\left(X-\langle X\rangle_{t, \varphi}\right)^{3}\right\rangle_{t, \varphi}}{\left\langle\left(X-\langle X\rangle_{t, \varphi}\right)^{2}\right\rangle_{t, \varphi}^{3 / 2}}$ ) and $\mathcal{K}=\frac{\left\langle\left(X-\langle X\rangle_{t, \varphi}\right)^{4}\right\rangle_{t, \varphi}}{\left\langle\left(X-\langle X\rangle_{t, \varphi}\right)^{2}\right\rangle_{t, \varphi}^{2}}-3$. 


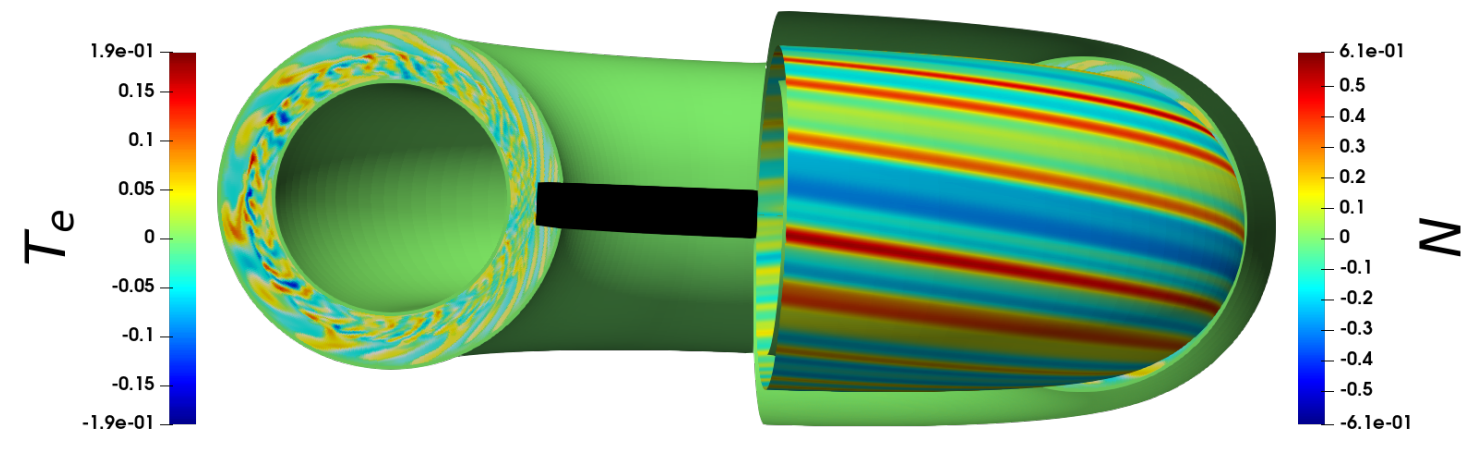

Figure 3: 3D snapshot of density and electron temperature fluctuations showing typical structures characteristics of edge plasma turbulence. The limiter is shown as thick black solid line for visual clarity. However in the actual simulations the limiter is taken as infinitely thin. Reference case, $\nu_{\star}=\nu_{\star, r e f}$.

The root mean square is evaluated as $\operatorname{rms}(X)=\left\langle\left(X-\langle X\rangle_{t, \varphi}\right)^{2}\right\rangle_{t, \varphi}^{1 / 2}$.

For the density, the shape of the PDFs is in agreement with the one observed in former isothermal studies [34. They evolve from being nearly Gaussian in the edge of the core region (roughly zero skewness and kurtosis) to being positively skewed in the SOL with a positive kurtosis indicating a heavy-tailed distribution. The skewness and the kurtosis sharply increase around the separatrix, underlining a strong change while crossing it. This indicates the transition from a behavior dominated by small amplitude events in the edge to a more intermittent regime dominated by the increasing appearance of large amplitude events in the SOL.

For the electron temperature, the PDFs remain nearly Gaussian in the edge of the core region as for the density. However, the small shift of the peak indicates a slight prevalence of small positive temperature events over the negative one (negative skewness, positive kurtosis). As for the density, the intermittency is preserved, and a sharp transition is seen across the separatrix, where PDFs move from being negatively to positively skewed. The negative kurtosis shows a tendency to enhance the presence of large rare temperature events into the SOL. Contrary to the density, comparisons of such behavior with experimental measurements are not straightforward due to the high level of incertitude on temperature fluctuations measurements, which are the results of indirect measurements [45] 48].

\subsection{Mean flow and equilibrium profiles}

The plasma equilibrium properties are investigated using mean flow variables averaged both on time and in the toroidal direction $\varphi$. By default, the HFS profiles are chosen right above the limiter location.

Fig. 5 shows the radial profiles of the density, electron and ion temperatures at both the high (HFS) and the low (LFS) field side midplane. The profiles are characteristic of a L-mode discharge without any transport barrier. Profiles on the HFS midplane show 


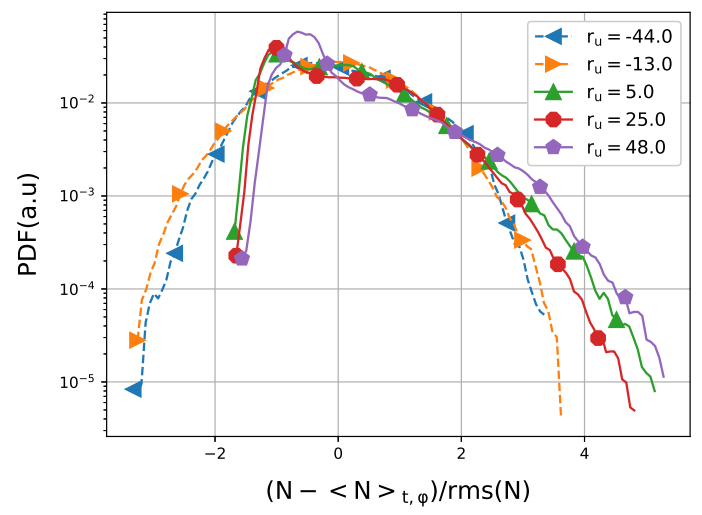

(a)

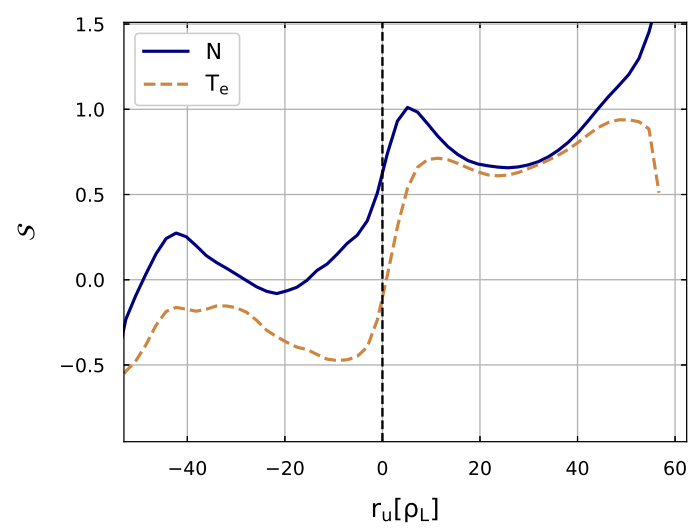

(c)

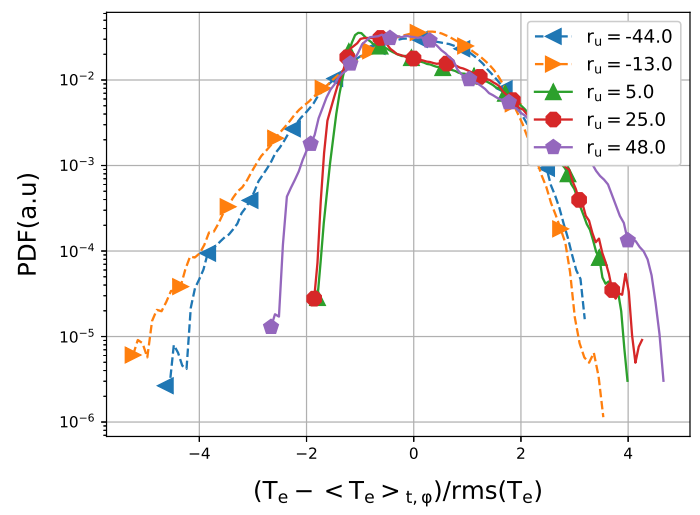

(b)

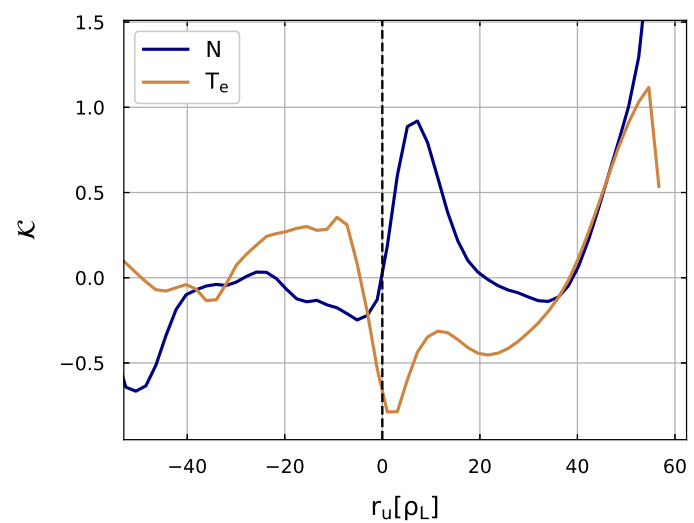

(d)

Figure 4: Turbulence fluctuations analysis. PDFs of density (a) and electron temperature (b) fluctuations at several radial locations on the LFS mid-plane. Profiles average in time and $\varphi$ direction of skewness (c) and kurtosis (d) at $\theta=0$ (LFS). The dashed lines corresponds to the separatrix. Reference case, $\nu_{\star}=\nu_{\star}, r e f$

steeper gradients at the separatrix than on the LFS, indicating a ballooned transport, i.e. a higher transport on the LFS than on the HFS. This feature is a well-known property of edge turbulence [49] caused by magnetic field inhomogeneity, leading to a poloidal asymmetry. Note finally that $T_{i}>T_{e}$, as expected from former experimental observations [1].

The poloidal asymmetry is still visible in Fig. 6. Density and electrons temperature profiles show indeed significant poloidal gradients leading to total pressure variations and large parallel flows. Due to the presence of the limiter, these variations are much larger into the SOL than in the edge of the core region with closed magnetic field lines. However, poloidal gradients of electronic temperature are slightly smaller than the density ones due to thermal conduction that only affects the heat transport. The occurrence of such large parallel flows has been already shown experimentally [49 51] and numerically [52, 53]. 


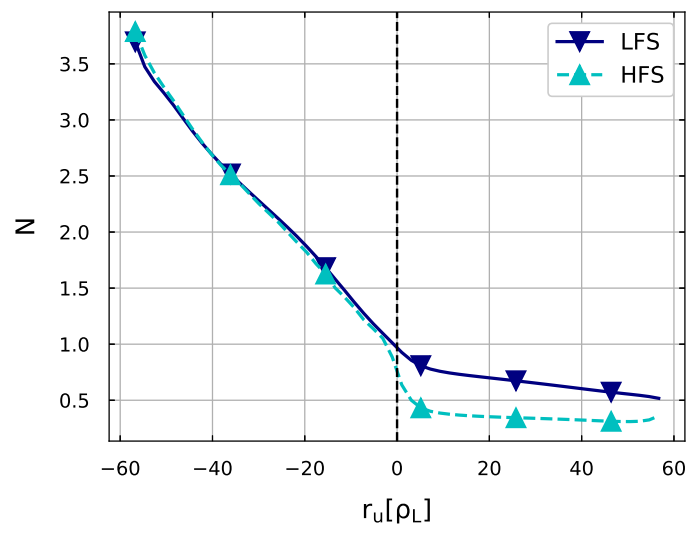

(a)

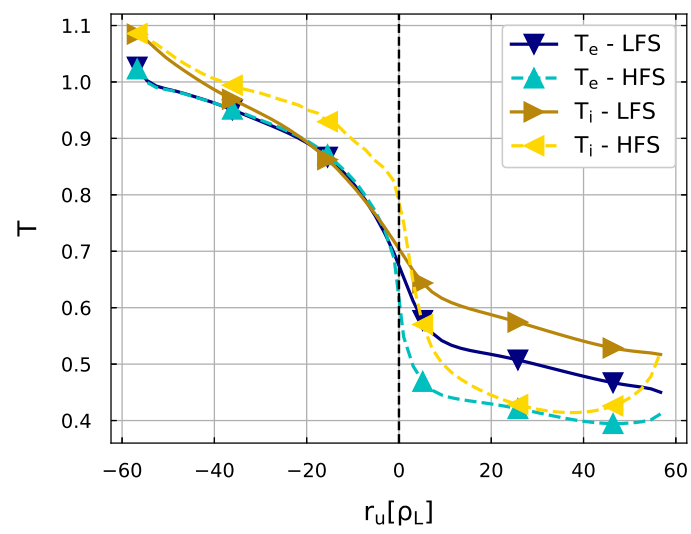

(b)

Figure 5: Radial profiles of density (a) and temperature (b) averaged in time and $\varphi$ direction at the LFS $(\theta=0)$ and HFS $(\theta=180)$ midplane. The dashed line corresponds to the separatrix. The rise on HFS of the temperature profiles is a numerical artefact due to the buffer region. Reference case, $\nu_{\star}=\nu_{\star}, r e f$.

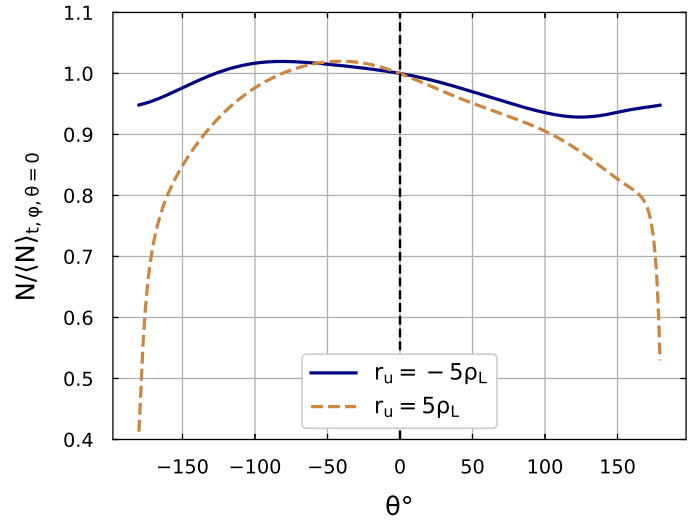

(a)

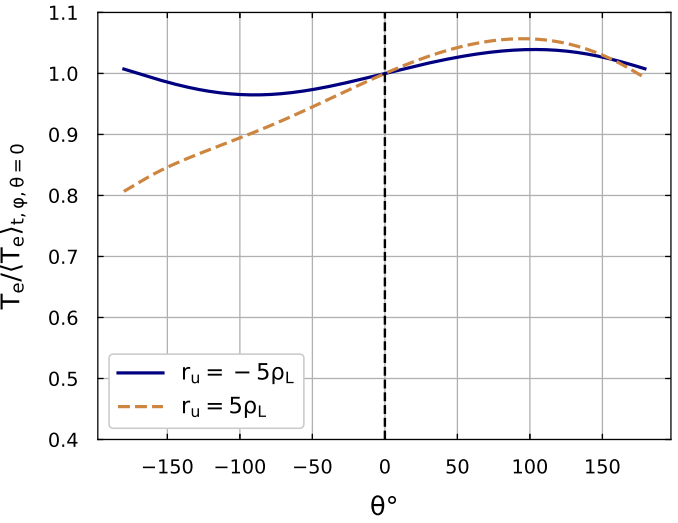

(b)

Figure 6: Poloidal profiles of density (a) and electrons temperature (b) in the edge of the core region (closed magnetic field lines (solid lines)) and into the SOL (open magnetic field line (dashed lines)). $\theta$ in degrees, increasing in the electron diamagnetic direction. Therefore $\theta=0^{\circ}$ corresponds to the LFS midplane, $\theta= \pm 90^{\circ}$ to the bottom and top of the machine, $\theta= \pm 180^{\circ}$ to the HFS midplane. Reference case, $\nu_{\star}=\nu_{\star, r e f}$.

\subsubsection{The SOL width}

The SOL width is a key parameter that determines the heat peak load on the plasma facing components. It can be determined from the radial profile of the SOL parallel heat flux at the target, defined here by $q_{\|}=N c_{s}\left(\gamma_{e} T_{e}+\gamma_{i} T_{i}\right)$, where $\gamma_{e / i}$ are the sheath heat transmission coefficients for electron and ion normally equal to 2.5 and 4.5. In this reference case, the solution exhibits a double exponential decrease as shown 
in Fig. 7. The radial profile of the parallel heat flux is well described by:

$$
q_{\|}=q_{n} \exp \left(-r_{u} / \lambda_{q, n}\right)+q_{f} \exp \left(-r_{u} / \lambda_{q, f}\right)
$$

$r_{u}$ being the distance from the separatrix. The fitting coefficients are $q_{n}=2.128$ and $q_{f}=3.842$. The squares and stars lines show the slope of the single exponential. It is worth mentioning that both density and temperature profiles exhibit this double e-folding length.

These results agree with recent works in limited plasma. Experimental measurements [54, 55] have shown the same kind of behavior for the decay length of the heat flux with a wider component $\left(\lambda_{q, f}\right)$ extended up to the wall and a shorter component $\left(\lambda_{q, n}\right)$ extended over few Larmor radii, namely the narrow feature. Such behavior has been also observed on density profiles with a two-layer structure showing a steep exponential decay near the separatrix and a much more gradual exponential decay in the far SOL[7, 8]. It is a critical aspect since it has been measured that the existence of $\lambda_{q, n}$ dramatically increases the heat flux on the target [56].

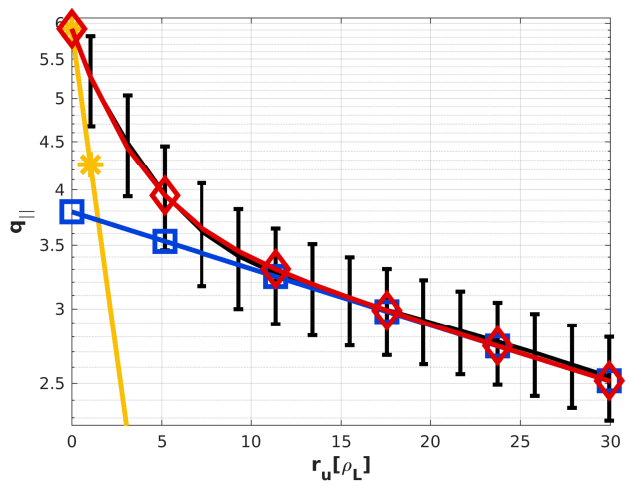

Figure 7: Parallel heat flux profile $q_{\|}$average in time and $\varphi$ direction (Eq. 7) in the SOL $\left(r_{u}>0\right)$ at the LFS midplane exhibiting a double exponential fitting. The squares and stars lines show the slope of the single exponential. The diamond curve is the result of the fitting. The black curve with error bars is the average profile and its standard deviation. $r_{u}$ is the distance from the separatrix. Reference case, $\nu_{\star}=\nu_{\star}, r e f$.

\subsection{Particles and heat transport}

The transport of a scalar quantity $X$ is carried out both by convection due to the drifts and by diffusion [57]:

$$
\Gamma_{X}^{\Psi}=u_{\perp} X-D_{\perp} \nabla_{\perp} X
$$

where $\Gamma^{\Psi}$ is the projection on the flux in the radial direction $\Psi, u_{\perp}$ is the perpendicular drift velocity defined in A.1, and $D$ is the diffusion coefficient. In order to evaluate the turbulent transport, scalar quantities are decomposed into a mean and a fluctuating part. Let's introduce a general average operator $\langle\cdot\rangle_{x_{i}}$ where 
$x_{i}=[t, \Psi, \theta, \varphi]$. Then it is possible to define for any scalar quantity $X$, its fluctuations part as $\tilde{X}=X-\langle X\rangle_{t, \varphi}$, where the averaging is performed both in time and the toroidal direction and $\langle\tilde{X}\rangle_{t, \varphi}=0$. The magnitude of the turbulent transport depends not only on the amplitude of the fluctuating transported quantities and their velocity, but as well as on the relative phase shifts (or cross phases) between them denoted $\delta_{\tilde{X}-\tilde{Y}}$. The estimate of $\delta$ performed in [58] for two general fluctuating fields having a Gaussian distribution with a zero mean value and a standard deviation equal to $\tilde{X}^{1 / 2}$ leading to:

$$
\cos \left(\delta_{\tilde{X}-\tilde{Y}}\right)=\frac{\langle\tilde{X} \tilde{Y}\rangle_{x_{i}}}{\sqrt{\left\langle\tilde{X}^{2}\right\rangle_{x_{i}} \cdot\left\langle\tilde{Y}^{2}\right\rangle_{x_{i}}}}
$$

The Eq. (9) determines the phase shift between $\tilde{N}$ and $\tilde{\Phi}$ and between $\tilde{T}_{e}$ and $\tilde{\Phi}$. They will be discussed in Sec. 5 .

\subsubsection{Radial particles flux}

According to Eq. (8), the total radial particles flux is $\Gamma_{i}^{\Psi}=N u_{\perp i}^{\Psi}-D_{\perp} \nabla_{\perp} N$ that leads to:

$$
\left\langle\Gamma_{i}^{\Psi}\right\rangle_{x_{i}}=\langle N\rangle_{x_{i}}\left\langle u_{E}^{\Psi}\right\rangle_{x_{i}}+\langle N\rangle_{x_{i}}\left\langle u_{\nabla B i}^{\Psi}\right\rangle_{x_{i}}+\left\langle\tilde{N} \tilde{u}_{\nabla B i}^{\Psi}\right\rangle_{x_{i}}+\left\langle\tilde{N} \tilde{u}_{E}^{\Psi}\right\rangle_{x_{i}}-D_{\perp} \nabla_{\perp}\langle N\rangle_{x_{i}}
$$

The first four terms on the RHS of Eq. 10 ) are respectively the average (mean flux) and fluctuating (turbulent flux) contributions related to the $E \times B$ and the ion curvature velocity drifts. In Fig. 8a, the relevant contributions of the particle flux are shown. Here we look at the total flux crossing a flux surface and averaged on time $\left\langle\iint \Gamma_{i}^{\Psi} \cdot d S\right\rangle_{t}$. These pictures look essentially the same as a LFS figure (which is not poloidally integrated) since most of the transport occurs on the LFS.

The averaged terms associated with the $E \times B$ velocity drifts are negligible in this case, and the fluctuating terms are almost entirely responsible for the particle transport. In agreement with former studies [59], the fluctuating terms of the $E \times B$ velocity drift carries out the total amount of particles, while the flux in the radial direction related to the ion perpendicular drift velocity is almost null. The diffusive flux that accounts for collisions and small scales turbulence is non-negligible in the core region, but it nearly vanishes into the SOL.

\subsubsection{Radial electron energy flux}

The total electron energy flux is defined as $\Gamma_{e}^{\Psi}=5 / 2 N T_{e} u_{\perp e}-\frac{3}{2} T_{e} D_{\perp} \nabla_{\perp} N-$ $N D_{\perp} \nabla_{\perp} T_{e}$. Its convective part can be decomposed into 10 terms involving mean 


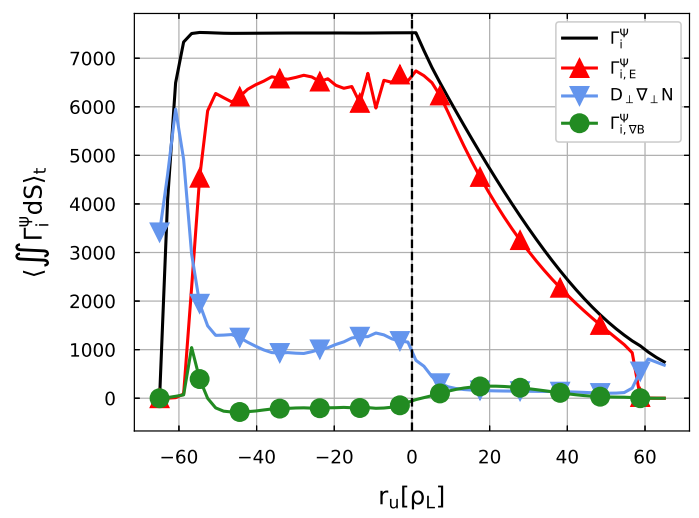

(a)

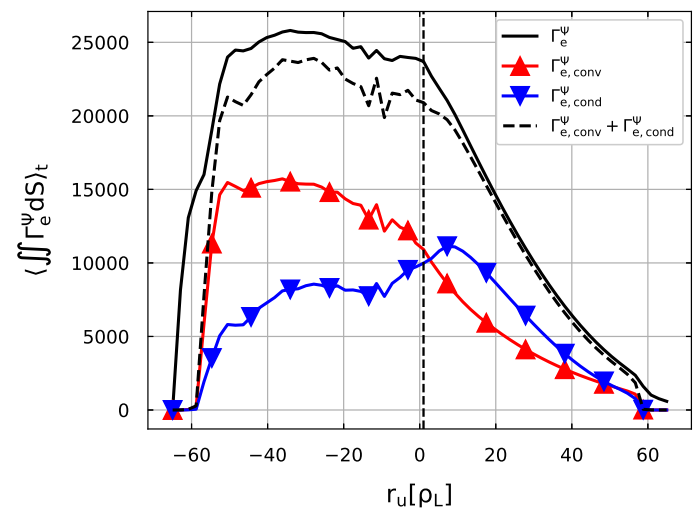

(b)

Figure 8: Profiles of various components of the particles (a) and electron energy (b) fluxes integrated over flux surfaces and averaged on time. The sharp variations of the profiles in the near vicinity of the radial boundaries are a numerical artefact due to buffer regions. $r_{u}$ is the distance from the separatrix. Reference case, $\nu_{\star}=\nu_{\star, \text { ref }}$.

quantities, double and triple correlations such that:

$$
\begin{aligned}
\left\langle\Gamma_{e}^{\Psi}\right\rangle_{x_{i}}=\frac{5}{2} & \left(\langle N\rangle_{x_{i}}\left\langle T_{e}\right\rangle_{x_{i}}\left\langle u_{E}^{\Psi}\right\rangle_{x_{i}}+\langle N\rangle_{x_{i}}\left\langle T_{e}\right\rangle_{x_{i}}\left\langle u_{\nabla B e}^{\Psi}\right\rangle_{x_{i}}+\left\langle\tilde{N} \tilde{T}_{e} \tilde{u}_{E}^{\Psi}\right\rangle_{x_{i}}+\left\langle\tilde{N} \tilde{T}_{e} \tilde{u}_{\nabla B e}^{\Psi}\right\rangle_{x_{i}}+\right. \\
& +\langle N\rangle_{x_{i}}\left\langle\tilde{T}_{e} \tilde{u}_{E}^{\Psi}\right\rangle_{x_{i}}+\left\langle u_{E}^{\Psi}\right\rangle_{x_{i}}\left\langle\tilde{N} \tilde{T}_{e}\right\rangle_{x_{i}}+\left\langle T_{e}\right\rangle_{x_{i}}\left\langle\tilde{N} \tilde{u}_{E}^{\Psi}\right\rangle_{x_{i}}+ \\
& \left.+\langle N\rangle_{x_{i}}\left\langle\tilde{T}_{e} \tilde{u}_{\nabla B e}^{\Psi}\right\rangle_{x_{i}}+\left\langle u_{\nabla B e}^{\Psi}\right\rangle_{x_{i}}\left\langle\tilde{N} \tilde{T}_{e}\right\rangle_{x_{i}}+\left\langle T_{e}\right\rangle_{x_{i}}\left\langle\tilde{N} \tilde{u}_{\nabla B e}^{\Psi}\right\rangle_{x_{i}}\right)+ \\
& -\frac{3}{2}\left\langle T_{e} D_{\perp} \nabla_{\perp} N\right\rangle_{x_{i}}-\left\langle N D_{\perp} \nabla_{\perp} T_{e}\right\rangle_{x_{i}}
\end{aligned}
$$

However, most of the terms in Eq.11 are trivial. The correlations between three fluctuating quantities are neglected. The major contribution can be reduced at only two terms as shown in Fig. 8b, which shows the radial distribution of the main components of the electron heat flux averaged over flux surfaces $\left(\left\langle\iint \Gamma_{e}^{\Psi} \cdot d S\right\rangle_{t}\right)$. In the edge of the core region, the electron heat transport is clearly dominated by the convection term $\left\langle T_{e}\right\rangle_{x_{i}}\left\langle\tilde{N} \tilde{u}_{E}^{\Psi}\right\rangle_{x_{i}}$ representing the energy associated to the $E \times B$ driven by turbulence. Instead, into the SOL, the heat conduction is the dominant mechanism. The conduction term $\langle N\rangle_{x_{i}}\left\langle\tilde{T}_{e} \tilde{u}_{E}^{\Psi}\right\rangle_{x_{i}}$ does not correspond to a transport of particles, but it is influenced by the fluctuations of $T_{e}$ that becomes in the near SOL comparable to the density fluctuations. As expected from earlier studies [4, 60], present results confirm that the heat conduction and convection induced by the $E \times B$ drift carry most of the heat.

\subsubsection{Poloidal variations of the radial fluxes}

Fig. 9 shows the poloidal distribution of particle, convective and conductive heat fluxes in the edge of the core region and in the SOL. As expected, the particles $E \times B$ 


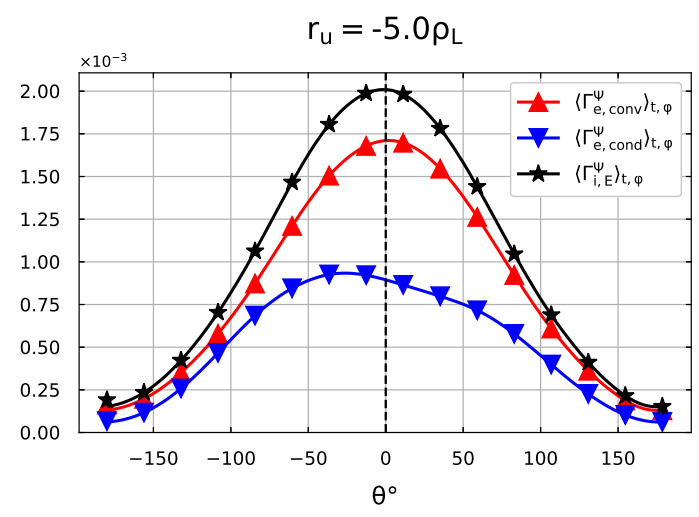

(a)

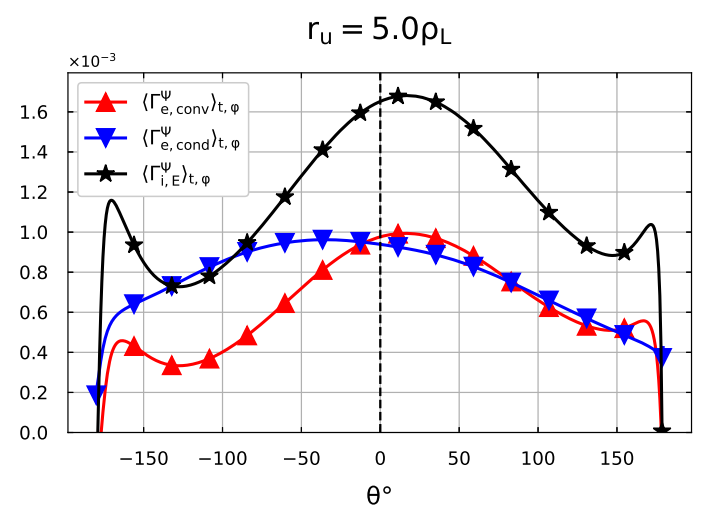

(b)

Figure 9: Poloidal profiles of the radial particles $E \times B$ flux, the conductive and the convective flux heat fluxes averaged in time and $\varphi$ direction. (a) $r_{u}=-10 \rho_{L}$ (closed field lines), and (b) $r_{u}=10 \rho_{L}$ (SOL). $\theta$ in degrees increasing in the electron diamagnetic drift direction. Reference case, $\nu_{\star}=\nu_{\star, r e f}$.

velocity drift flux and the heat convective flux show the same trend. Strongly peaked in an angular sector around $\theta=0^{\circ}$, they confirm the signature of a ballooned transport in the edge of the core region, with the presence of non-symmetric large amplitude parallel flows at the outboard midplane (LFS). Comparatively, the conductive heat is flatter because the parallel heat conduction reduces the poloidal (LFS/HFS) asymmetries on $T_{e}$ fluctuations. In the SOL, the ballooning of the flux is reduced. The higher temperature gradient leads to an increased contribution of the conduction. It is worth noticing that additional local peaks occur in the SOL close to the limiter due to perturbations induced in the plasma.

\section{Impact of collisionality on flow properties}

Simulations have been performed in the range of collisionality values between 0.3 and $1000 \nu_{\star, r e f}$. All values of $\nu_{\star}$ and corresponding $\eta_{\| 0}$ and $\chi_{\| 0 e / i}$ are given in Tab 2. As mentioned in Sec. 3, $\nu_{\star} \geq 100 \nu_{\star}$, ref are artificially high values, in the sense that they are much higher than realistic values of experiments. They allow stressing the impact of high collisionality on the plasma properties. Besides, numerical results show that solutions behave differently at these two high values. For the smallest collisionality value $\nu_{\star}=0.3 \nu_{\star, r e f}$, a simulation has been also performed with a time and space-dependent parallel resistivity, $\eta_{\|}\left(T_{e}^{-3 / 2}\right)$ (Eq.6). However, the following results only show a small impact with respect to the solution obtained with constant $\eta_{\|}$at the same collisionality. The impact of varying the collisionality is firstly analyzed on turbulence through the edge and SOL fluctuations, then on equilibrium profiles and mean flow properties. 


\subsection{Edge and SOL fluctuations}

\subsubsection{Impact on the fluctuations amplitude}

The amplitude of fluctuations is a measure of the level of turbulence energy. Fig 10 shows the radial profiles of the relative fluctuations level of density and electron temperature $\left(r m s(N) / N, r m s\left(T_{e}\right) / T_{e}\right)$ at the LFS midplane. In agreement with experiments, the fluctuation levels of temperature are usually smaller than for the density at the same collisionality [61, 62].

In the edge of the core region, the fluctuations levels increase with the radius at the LFS midplane and steepen at the separatrix for all values of $\nu_{\star}$ smaller than $10 \nu_{\star r e f}$ in agreement with experiments [5, 63]. The maxima are reached right across the separatrix into the SOL region. When decreasing the collisionality, the fluctuations level for both density and temperature decreases in the core, while into the SOL, it is nearly insensitive for the density but increases for the temperature fluctuations. Such behavior for the temperature fluctuations into the SOL suggests an additional instability mechanism injecting energy into the system when lowering the collisionality value. A candidate could be the sheath-driven conductive wall instability (SCW), which is a drift-wave like instability for the temperature, and thus it should exhibit an increasing intensity when collisionality has a small non-zero value [64 68]. The coupling mechanism between the plasma and this instability is related to a positive feedback loop between the driving temperature fluctuations $\tilde{T}_{e}$, the parallel current fluctuations $\tilde{j}_{\|}$and the electric potential fluctuations $\tilde{\Phi}$ through the Bohm boundary condition Eq.1 and the Ohm's Law (Eq. A.6). Lowering the collisionality increases the values of $\mathrm{rms}\left(T_{e}\right) / T_{e}$, and thus increases the level of energy.

Differently, for the two high collisionality values $\nu_{\star}=100,1000 \nu_{\star}$, ref , the variations of the fluctuations level over the radius is weak for both the density and the temperature with relatively flat profiles. Moreover, in the edge of the core region (closed field lines) and in the SOL (open field lines) as well, the fluctuations level globally decreases when the collisionality increases.

\subsubsection{Impact on the probability density functions}

The impact of varying collisionality on turbulence statistical properties is analyzed here from the PDFs of density and temperature, Fig. 11.

No major change is observed when changing collisionality compared to the reference case for $\nu_{\star} \leq 10 \nu_{\star}$ ref. The near-zero skewness in the edge of the core region for every collisionality value indicates Gaussian-like shape PDFs (not shown here) that evolve rapidly across the separatrix to positively skewed PDFs into the SOL, holding their intermittent signature. PDFs into the SOL shrink when lowering the collisionality (smaller skewness), meaning the amplitude of high density and temperature events reduce with $\nu_{\star}$. 


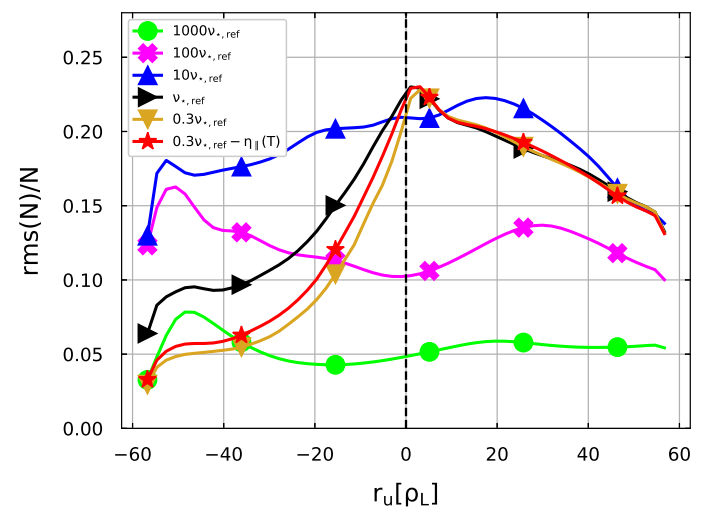

(a)

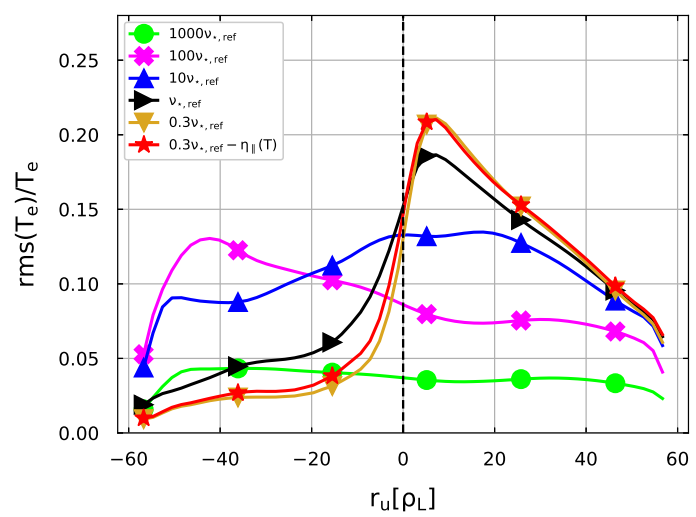

(b)

Figure 10: Radial profiles of the density (a) and electron temperature (b) fluctuations level averaged in time and $\varphi$, at the LFS midplane and depending on the collisionality $\nu_{\star}$. The sharp variations of the profiles at the radial boundary is an artifact related to the numerical buffer regions introduced in Sec.2.

At the two high collisionalities $\nu_{\star}=100,1000 \nu_{\star}$, ref , the skewness acts in a completely different way with respect to the reference case. PDFs evolve from positively skewed PDFs into the edge of the core region to negatively skewed ones into the SOL, except for the temperature at $\nu_{\star}=100 \nu_{\star}$, ref where the PDF remains positively skewed along the radius. Besides, the PDFs show a symmetric distribution with a double peak (same amplitude positive and negative event equally probable). A possible explanation might be correlated with the fact that turbulent structures at very high collisionality are no longer electrically connected with the target causing a different redistribution of their amplitude [15, 69]. Also, as described in the next section, the size of coherent structures increasing with collisionality, the turbulence structures spread over both open and closed field lines simultaneously.

\subsubsection{Impact on the coherent structures size}

The impact of changing collisionality on the size of the coherent structures is of primary importance because this can change transport quantity as $\tilde{u}_{E}^{\Psi} \simeq-k_{\theta} \tilde{\Phi}$. Results in Fig. 12 show that turbulence structures remain aligned along the magnetic field lines as already shown for the reference case on Fig. 3. When decreasing the collisionality from $1000 \nu_{\star, \text { ref }}$ to $0.3 \nu_{\star, \text { ref }}$, the size of the structures is strongly reduced, meaning that $k_{\|} \simeq k_{\varphi}$ and $k_{\perp} \simeq k_{\theta}$ are increasing. This is in agreement with studies on filamentary structures [9, 70] which have shown the same trend. We can note that the radial expansion of turbulence structures follows the same trend.

\subsubsection{Impact on the phase shift}




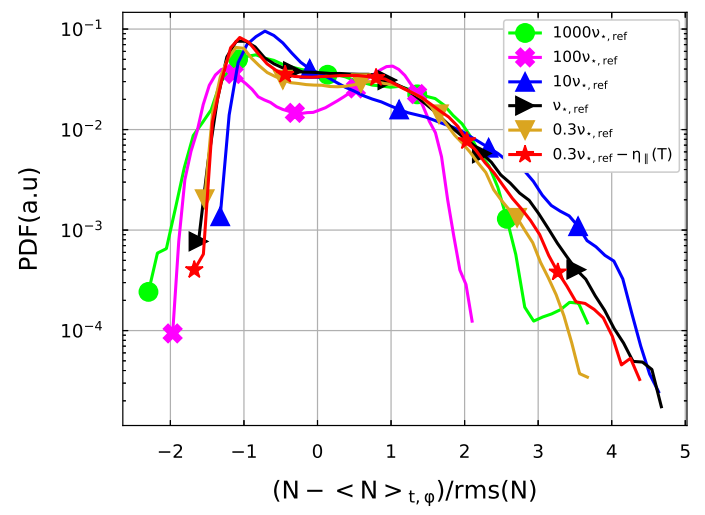

(a)

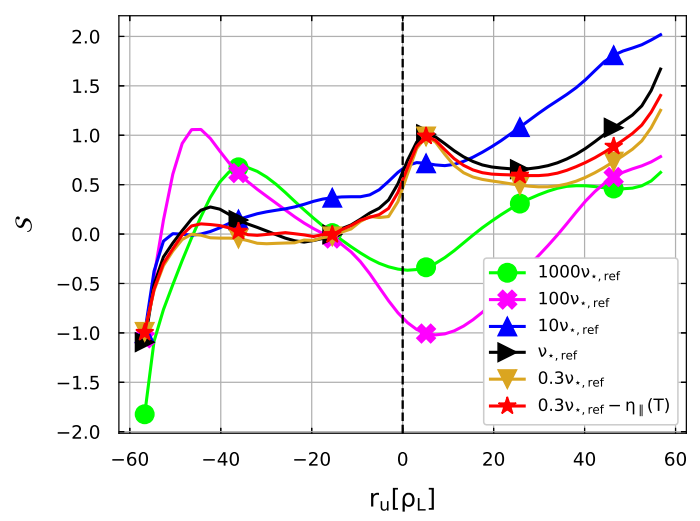

(c)

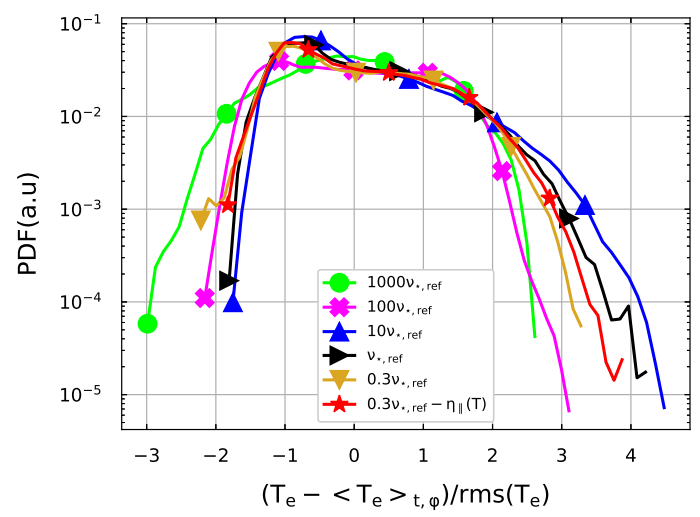

(b)

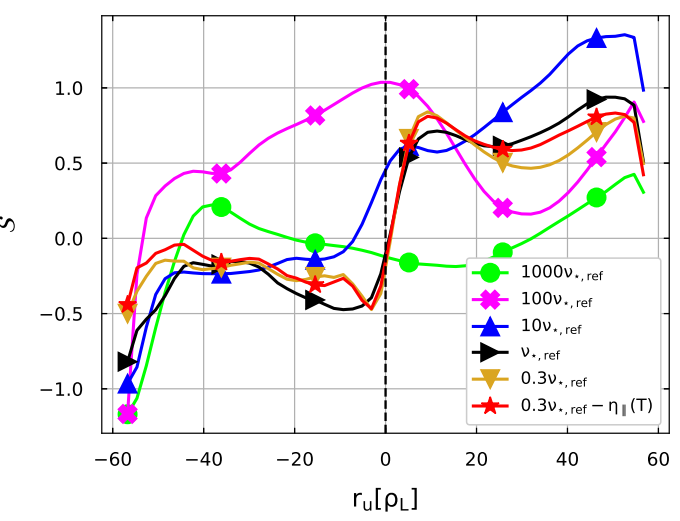

(d)

Figure 11: PDFs of density and temperature fluctuations into the SOL at $r_{u}=30 \rho_{L}$ depending on the collisionality $(\mathrm{a}, \mathrm{b})$. Profiles of density and temperature skewness averaged in time and $\varphi$ direction depending on the collisionality $(c, d)$.
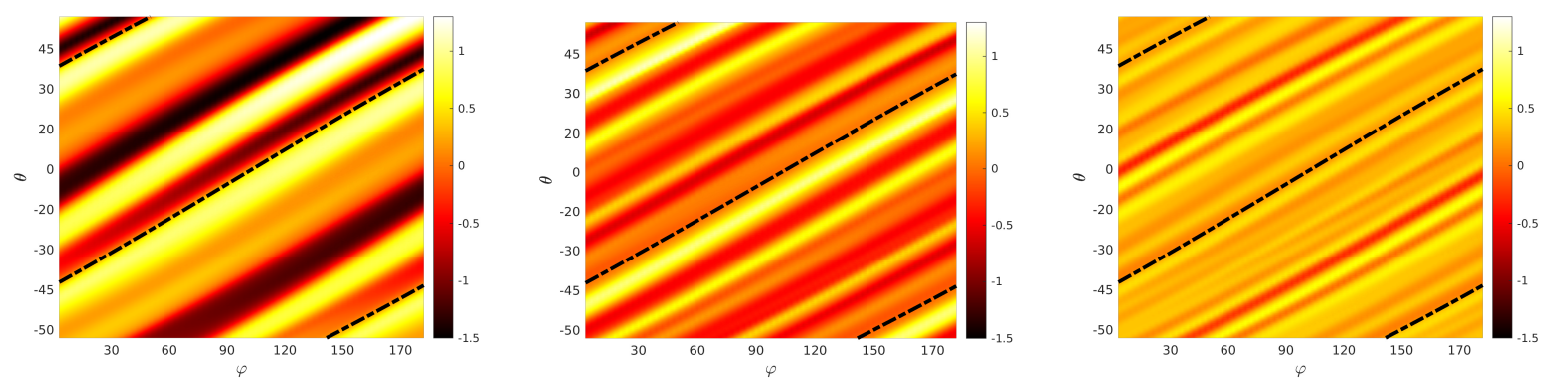

Figure 12: $2 \mathrm{D}$ maps of electrostatic potential $\tilde{\Phi}$ over flux surfaces at $r_{u}=-10 \rho_{L}$ (closed magnetic field lines region), zoom in on LFS. From left to right $\nu_{\star}=1000,10,0.3 \nu_{\star}$ ref . Black dashed lines are the magnetic field lines. 
As mentioned in Sec. 4, the magnitude of particles and energy fluxes also depends on the phase shifts between fluctuations of the electric potential and plasma density and the electric potential and electron temperature. Moreover, the phase shifts between the electric potential and plasma density fluctuations guide the identification of the instabilities that drive turbulence at the plasma edge. In particular, a phase shift close to $\pi / 2$ corresponds to the linear interchange instability, while a phase shift smaller than $\pi / 4$ is typical of the linear drift wave instability [17]. These theoretical results are rigorously valid in linear regimes, the information provided cannot be considered fully conclusive in the nonlinear regimes investigated in the present simulations.

Tab3 reports the values measured in the simulations. In the edge of the core region, present results show a reduction of the phase shifts when lowering the collisionality value. This could suggest that at low collisionality, the main drive of turbulence changes from the interchange to the drift wave instability. For the collisionality of the reference case and smaller $\left(\nu_{\star}=\nu_{\star, \text { ref }}\right.$ and $\left.\nu_{\star}=0.3 \nu_{\star, \text { ref }}\right), \delta_{\tilde{N}-\tilde{\Phi}}$ is about $\pi / 4$. Into the SOL, the reduction is less marked due to the dominance of the sheath resistance $R_{\text {sheath }}$, which is collisionality independent, on the parallel resistance $R_{\|}$. This suggests that the interchange instability remains stronger into the SOL regardless of the collisionality value as already reported in gyro-fluid simulations in [71]. On the other side for higher collisionalities $\left(\nu_{\star}>10 \nu_{\star, \text { ref }}\right)$ the phase shift is closer to $\pi / 2$ in both the edge core region and into the SOL, underlining the predominance of the interchange mechanism at high collisionalities in the whole simulation domain.

Finally, the decreasing value of $\delta_{\tilde{T}_{e}-\tilde{\Phi}}$ when lowering the collisionality value in the edge of the core region can be related to the property of the electrons to become more and more adiabatic. In this case, from the Ohm law, we can expect that the phase between the electric potential and the density and the electron temperature reduces.

\begin{tabular}{|c|c|c|c|c|}
\hline & \multicolumn{2}{|c|}{$\delta_{\tilde{N}-\tilde{\Phi}}$} & \multicolumn{2}{c|}{$\delta_{\tilde{T}_{e}-\tilde{\Phi}}$} \\
& EDGE & SOL & EDGE & SOL \\
\hline $1000 \nu_{\star, \text { ref }}$ & $0.46 \pi$ & $0.43 \pi$ & $0.58 \pi$ & $0.63 \pi$ \\
$100 \nu_{\star, \text { ref }}$ & $0.42 \pi$ & $0.37 \pi$ & $0.67 \pi$ & $0.58 \pi$ \\
$10 \nu_{\star, \text { ref }}$ & $0.36 \pi$ & $0.34 \pi$ & $0.5 \pi$ & $0.49 \pi$ \\
$\nu_{\star, \text { ref }}$ & $0.27 \pi$ & $0.35 \pi$ & $0.32 \pi$ & $0.47 \pi$ \\
$0.3 \nu_{\star, \text { ref }}$ & $0.24 \pi$ & $0.35 \pi$ & $0.27 \pi$ & $0.47 \pi$ \\
$0.3 \nu_{\star, \text { ref }}(T)$ & $0.23 \pi$ & $0.34 \pi$ & $0.27 \pi$ & $0.47 \pi$ \\
\hline
\end{tabular}

Table 3: Values of the post-treated relative potential-density $\delta_{\tilde{N}}-\tilde{\Phi}$ and potential-temperature $\delta_{\tilde{T}_{e}-\tilde{\Phi}}$ phase shifts when changing $\nu_{\star}$. The reference case is highlighted.

\subsection{Equilibrium profiles and radial fluxes}

The complex interactions between large-scale fluctuations described above, microturbulence, and mean flow leads to investigate the impact of the collisionality on the 
equilibrium profiles and the transport properties. Fig. 13 shows the time-toroidal averaged profiles of the density, the electrostatic potential, and the electron and ion temperatures at the LFS midplane. Results show that generally lowering the collisionality increases the radial gradient of all fields across the separatrix excepted for $\nu_{\star}=100,1000 \nu_{\star, \text { ref }}$ cases for which the radial profiles are globally flat.

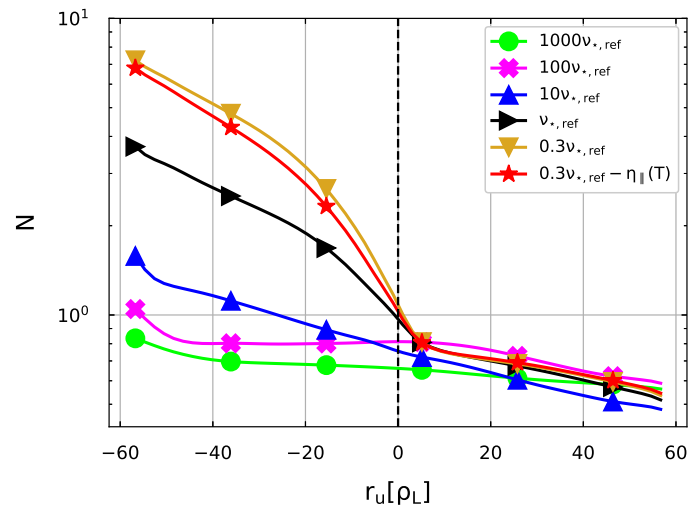

(a)

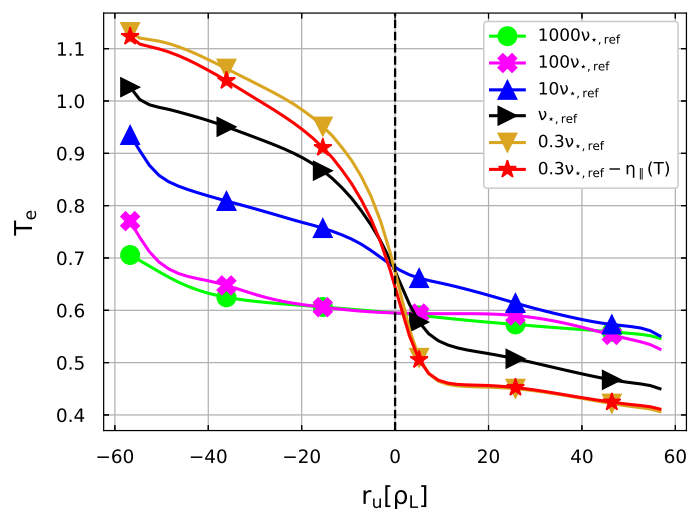

(c)

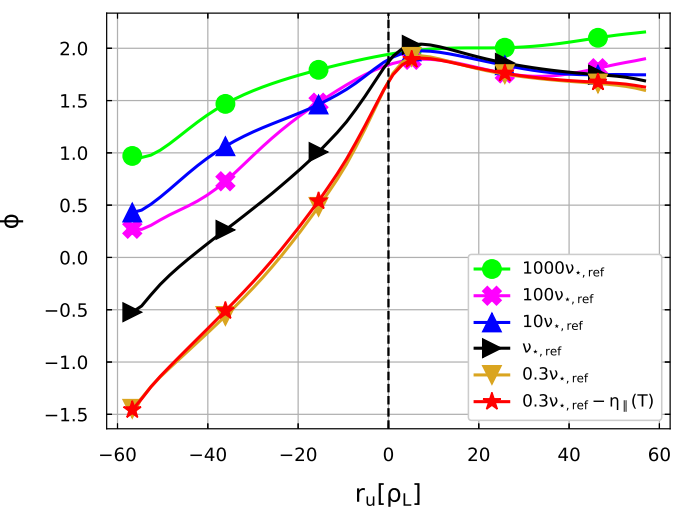

(b)

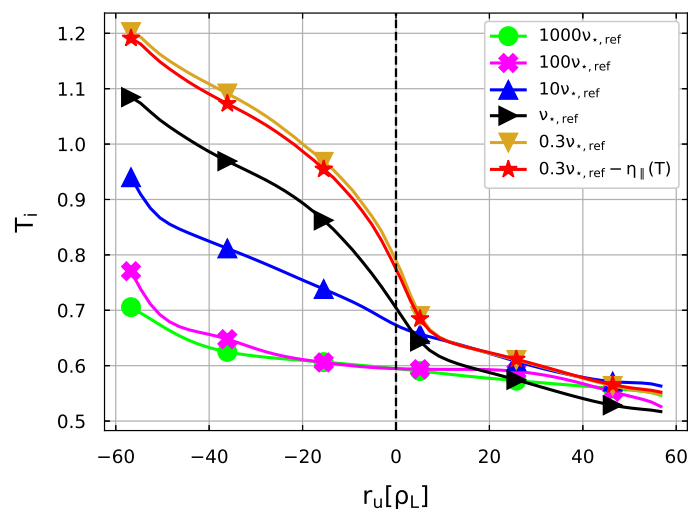

(d)

Figure 13: Radial profiles of the density $N$ (a), the electrostatic potential $\Phi($ b), the electron $T_{e}(\mathrm{c})$ and the ion $T_{i}(\mathrm{~d})$ temperature averaged in time and $\varphi$ direction at the LFS midplane depending on the collisionality. The dashed line shows the separatrix.

The density profiles in the core-edge (Fig. 13a) steepen when lowering $\nu_{\star}$ that leads to an increase of the diffusive particles flux. Since the imposed total flux is constant in such simulations, the convective transport is reduced by decreasing collisionality value, Fig. 15a. This is in agreement with the lower density fluctuations level and the lower value of $\delta_{\tilde{N}-\tilde{\Phi}}$ when $\nu_{\star}<\nu_{\star, \text { ref }}$ as seen above. Indeed when $\nu_{\star}$ is equal to the reference case collisionality or larger, the turbulent $E \times B$ transport still increases mostly because the phase shift rises up, and even if $r m s(N) / N$ goes down. In the case where $\nu_{\star}=1000 \nu_{\star, r e f}$, although the phase shift reaches its maximum, the amplitude of fluctuations is so low that they are no longer able to provide any contribution to the radial particle flux. Instead, it is the mean-field transport that drives $\Gamma_{i}^{\Psi}$. Into the $\mathrm{SOL}$, the mean density profiles flatten with increasing $\nu_{\star}$ as already reported in many 
experiments in tokamaks [72 75]. Here the profiles seem to be mostly independent of the collisionality except in the near SOL. This might be due to the fact that no matter what the collisionality is, the effective value of the resistance is always big enough to prevent the appearance of any gradients. Indeed for $\nu_{\star} \leq \nu_{\star, r e f}$ the sheath resistance overwhelm the parallel one Appendix B. Meanwhile when $\nu_{\star} \geq 10 \nu_{\star}$ the parallel resistance raises up to be a bigger than $R_{\text {sheath }}$. At this point, $R_{\|}$dominates the dynamic into the SOL, but its value is so high that the resulting profile remains flat. Recent analyses have also suggested a link between the SOL profiles and the filaments dynamics [76]. The reduction of the turbulent particle transport could be interpreted as a consequence of the effect of the $E \times B$ shear on the dynamics of turbulent eddies [77-79]. However, results in Fig $15 \mathrm{~b}$ show that the increase of the $E \times B$ shear with collisionality is only localized around the separatrix while the reduction of the turbulent particle transport is global over the radius. At most, the increasing shear could explain the small pits into the profiles observed at the separatrix on Fig. 15a for $\nu_{\star}<\nu_{\star}$,ref.

The value of the shear depends on the $\Phi$-radial profile (Fig.13b). The electrostatic potential presents a maximum right after the separatrix and two different concavities in the core-edge region and the SOL. That leads to two opposite contributions of $u_{E}^{\theta}$ (negative and positive), thus increasing the overall shear between the two regions. The gradients of the electrostatic potential in the edge of the core region are the same as the ion pressure gradients $\nabla \Phi \simeq-\nabla P_{i}$, and they decrease when increasing the collisionality value. Into the $\mathrm{SOL}$, the relation $\Phi \simeq \Lambda T_{e}$ is always invoked to estimate the radial electric field $\left(E_{\Psi} \simeq-3 \partial_{\Psi} T_{e}\right)$. Since electron temperature profiles into the SOL vary with collisionality (Fig $13 \mathrm{c}$ ), one could expect the profiles of electrostatic potential to behave in the same way. However, results show that into the SOL the electrostatic potential always decreases when $\nu_{\star} \leq 10 \nu_{\star}$ ref . It reaches a value independent of $\nu_{\star}$, whereas a slight increase in the far SOL is observed for $\nu_{\star} \geq 100 \nu_{\star}$ ref . Therefore, although the relation $\Phi \simeq \Lambda T_{e}$ is a good approximation at the HFS midplane, as shown in $14 \mathrm{a}$ and $14 \mathrm{~b}$, it does not well describe the situation for both higher and lower collisionality values than $\nu_{\star, r e f}$ at the LFS midplane. The reason for this discrepancy is due to the presence of non-null parallel gradients of density and temperature, respectively on Fig $14 \mathrm{c}$ and $14 \mathrm{~d}$. In the case where $\nu_{\star} \leq \nu_{\star}$ ref the temperature poloidal profiles are almost flat with nearly zero gradients. On the contrary, the density profiles show quite large variations at all collisionality values. For $\nu_{\star} \geq 10 \nu_{\star}$ ref a strong increase in the parallel gradient of $T_{e}$ is observed. This could partially explain the increment of the electrostatic potential at the highest collisionality value. These results agree with the recent theoretical work of [80] which shows that to estimate the radial electric field, we need to take into account not only the value at the sheath but the local values of the density and the temperature.

Focusing now on temperature profiles, results show that $T_{e}$ and $T_{i}$ behave quite similarly with temperatures decreasing with the radius. For $\nu_{\star}<10 \nu_{\star}$,ref , the radial gradients remain nearly insensitive to the collisionality values in the edge of the closed field lines region and the far SOL. However, near the separatrix, the gradients increase 


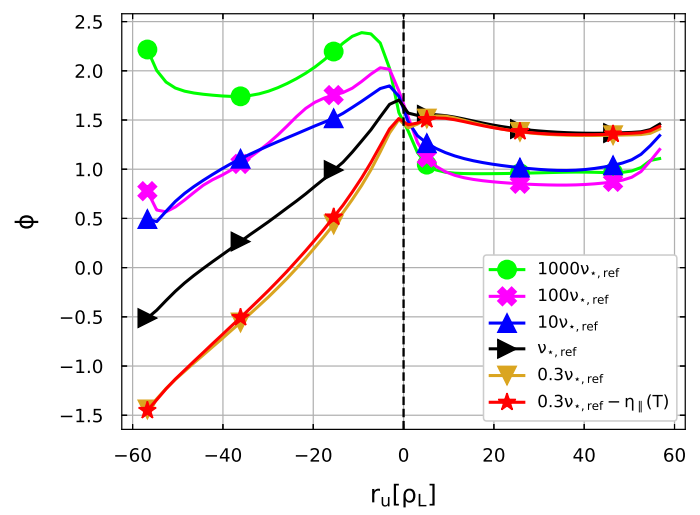

(a)

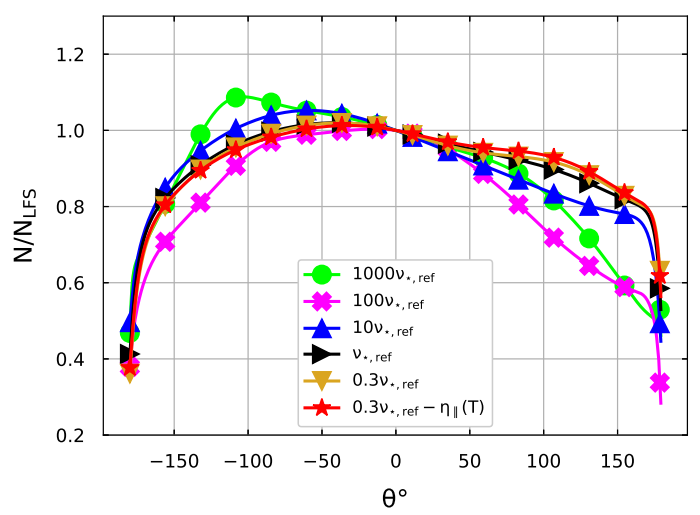

(c)

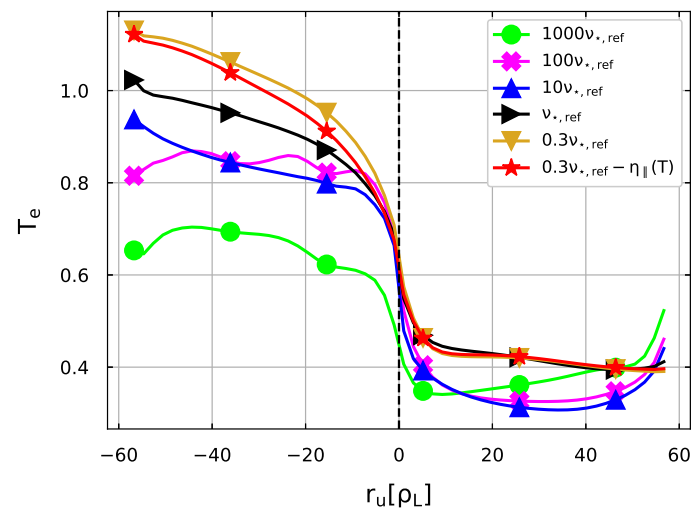

(b)

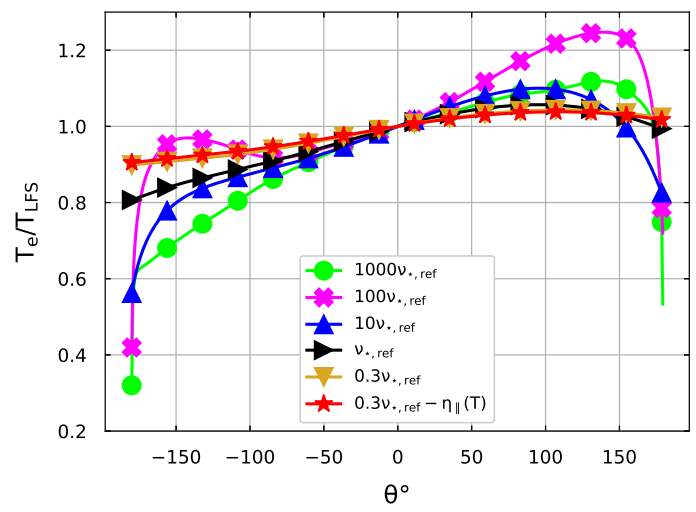

(d)

Figure 14: Radial profiles of the electrostatic potential $\Phi$ (a) and the electron temperature $T_{e}$ (b) averaged in time and $\varphi$ direction at the HFS midplane depending on $\nu_{\star}$. Poloidal profiles of $N(\mathrm{c})$ and $T_{e}(\mathrm{~d})$ averaged in time and $\varphi$ direction in the SOL at $r_{u}=5 \rho_{L}$ depending on $\nu_{\star}$.

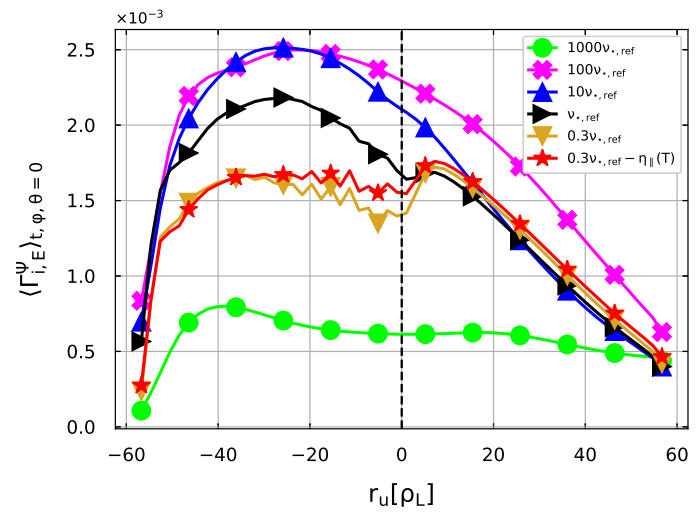

(a)

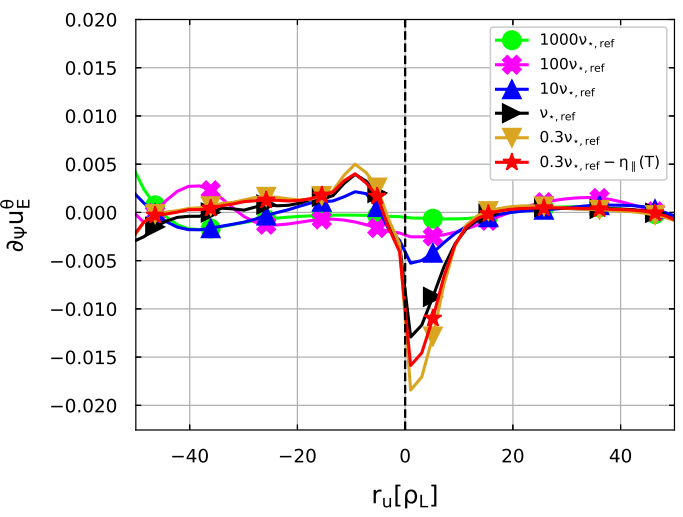

(b)

Figure 15: Radial profiles of the turbulent particles radial flux $\left(\left\langle\tilde{N} \tilde{u}_{E}^{\Psi}\right\rangle_{t, \varphi}\right)$ (a) and of the $E \times B$ shear $\left(\left\langle\partial_{\Psi} u_{E}^{\theta}\right\rangle_{t, \varphi}\right)$ (b) averaged in time and $\varphi$ direction at the LFS midplane depending on the collisionality. 
when the collisionality value is decreased. The electron temperature keeps the same value at the separatrix, meanwhile when decreasing the collisionality value, $T_{e}$ increases in the edge and decreases in the SOL. For the ion temperature, the value at the separatrix changes, $T_{i}$ increases in the core-edge while the temperature in the SOL remains roughly unchanged. For the two high values of the collisionality, $\nu_{\star} \geq 100 \nu_{\star}$, ref , the profiles of $T_{i}$ are approximately flat over the whole radius.

The variation of the radial energy flux with collisionality values at LFS and HFS is shown in Fig $16 \mathrm{a}$ and $16 \mathrm{~b}$.

The respective part in percent of each component of the total flux is shown at four radial locations, two before and two after the separatrix. The fluxes are normalized to their total value. Contribution lower than the $3 \%$ are merged into subscript "other contr."

In Fig $16 \mathrm{a}$ we can observe that except for the very high collisionality $\left(\nu_{\star}=\right.$ $\left.1000 \nu_{\star, r e f}\right)$, where the mean contribution $\left\langle N T_{e} u_{E}^{\Psi}\right\rangle_{t, \varphi}$ is prevailing over the other as it was for the particle flux, convection $\left\langle T_{e}\right\rangle_{t, \varphi}\left\langle\tilde{N} \tilde{u}_{E}^{\Psi}\right\rangle_{t, \varphi}$ and conduction $\left(\langle N\rangle_{t, \varphi}\left\langle\tilde{T}_{e} \tilde{u}_{E}^{\Psi}\right\rangle_{t, \varphi}\right)$ are largely dominant whatever the collisionality values. Convection heat flux dominates in the edge of the core region in all simulations. The high temperature fluctuations at low collisionality values contribute to increase the heat conduction into the SOL at a level where it becomes comparable and even higher than the convective contribution. Indeed, after the separatrix the thermal conductivity decreases, and therefore the gradient of temperature becomes much stronger. This leads to a transport of heat without transport of particles. In the edge of the core region, an increasing diffusive contribution is observed $\left(\left\langle D_{\perp} \frac{3}{2} T_{e} \nabla N\right\rangle_{t, \varphi}\right)$ when lowering the collisionality for $\nu_{\star} \leq \nu_{\star, \text { ref }}$ due to the existence of higher density gradients. A third order contribution $\left(\left\langle\tilde{N} \tilde{T}_{e} \tilde{u}_{E}^{\Psi}\right\rangle_{t, \varphi}\right)$ is active into the SOL associated with larger fluctuations found when $\nu_{\star}$ is below $\nu_{\star}, r e f$. At HFS (Fig 16b) in the core-edge region the contribution given by the curvature drift $u_{\nabla B, e}^{\Psi}$ arise compared to LFS in all the whole range of analyzed collisionality. However, this is much stronger at low collisionality. For $\nu_{\star} \geq 10 \nu_{\star, r e f}$ the curvature is counterbalanced mostly by the heat convection. Meanwhile, when $\nu_{\star}=1000 \nu_{\star}$,ref the contribution of the average electric drift is always prevalent at all radial location, except right after the separatrix. The presence of strong curvature drift has been suggested to induce the development of parallel Kelvin-Helmholtz instability as reported in [81, 82]. In closed field line conduction heat, it seems to play a minor role, whereas its importance increases in the near SOL. Diffusive term seems to be relevant at $\nu_{\star} \leq \nu_{\star, \text { ref }}$, whereas a small contribution due to solely fluctuations arise across the separatrix for $\nu_{\star} \leq \nu_{\star, \text { ref }}$

\subsection{SOL width}

The change of $N$ and $T_{e, i}$ profiles with $\nu_{\star}$ has a direct impact on the parallel heat fluxes. Experimentally the presence of the narrow feature in limited plasma has been found to be very sensitive at the collisionality changes [54]. The narrow feature present in the reference case at $\nu_{\star, \text { ref }}$ remains when decreasing the collisionality to $0.3 \nu_{\star, r e f}$, Fig. 17a. 


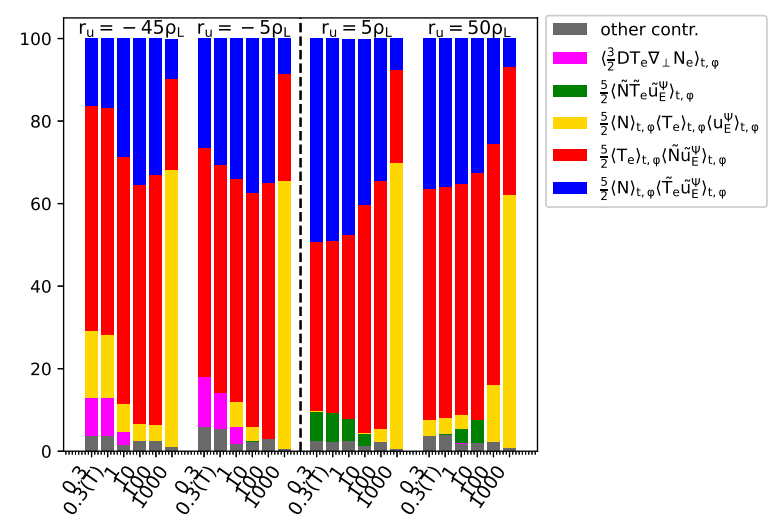

$\nu_{*}$, ref

(a)

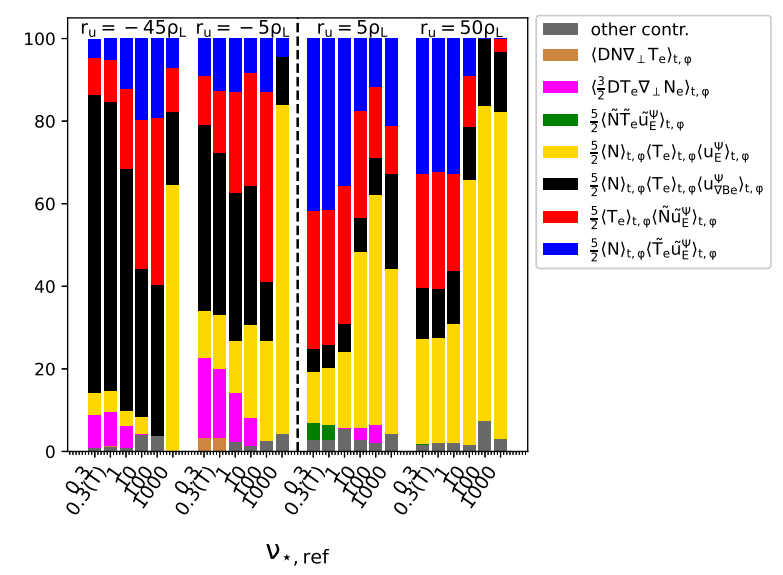

(b)

Figure 16: Heat flux components distribution in percent of the total flux at $\theta=0^{\circ}$ (Fig 16a) and $\theta=180^{\circ}$ (Fig $16 \mathrm{~b}$ at four radial locations and depending on the collisionality.

However, this is no longer true when increasing the collisionality to $10 \nu_{\star}$ ref for which the fit with a single exponential becomes an excellent approximation, Fig. 17b,

To complete our analysis, the evaluations of $\lambda_{q, n}$ and $\lambda_{q, f}$ with respect to $\nu_{\star}$ are shown on Fig 18. Results show that both near and far SOL widths increase when increasing the collisionality value. This enlargement of the SOL width with collisionality is in agreement with former experimental studies in [15]. The amplitude of the narrow feature increases when $\nu_{\star}$ decreases, as demonstrated by an lower ratio $q_{n} / q_{f}$ at $\nu_{\star, r e f}$ than at $0.3 \nu_{\star}$ ref with values equal to about 0.5 and1.1, respectively. The decrease of the narrow feature strength $q_{n} / q_{f}$ with increasing $\nu_{\star}$, eventually resulting in its disappearance, is consistent with previous experimental results [54]. Furthermore, $q_{n} / g_{f}$ correlates well with the value of the EXB shear close to the LCFS (Fig 15b), which, according to the theoretical model from [83], is the primary cause of its formation. Moreover we can investigate the relative contribution that ion and electrons give singularly to $q_{\|}$in Fig. $17 \mathrm{c}$ and $17 \mathrm{~d}$ for $\nu_{\star}=0.3,10 \nu_{\star}$,ref respectively. The percentage refers to the value of $q_{\|}$at the separatrix. The part associated with the electrons is 


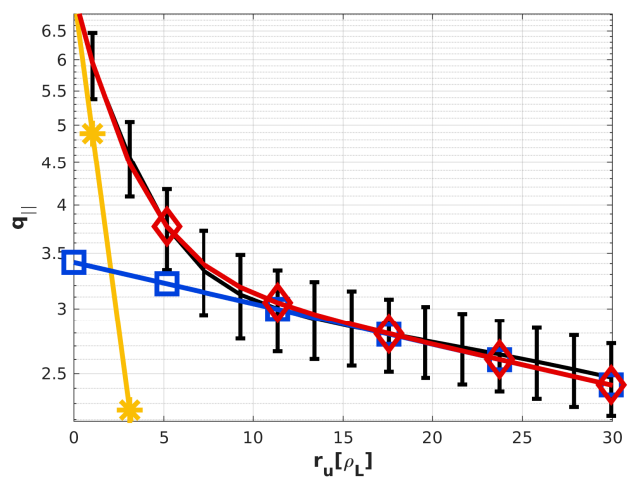

(a)

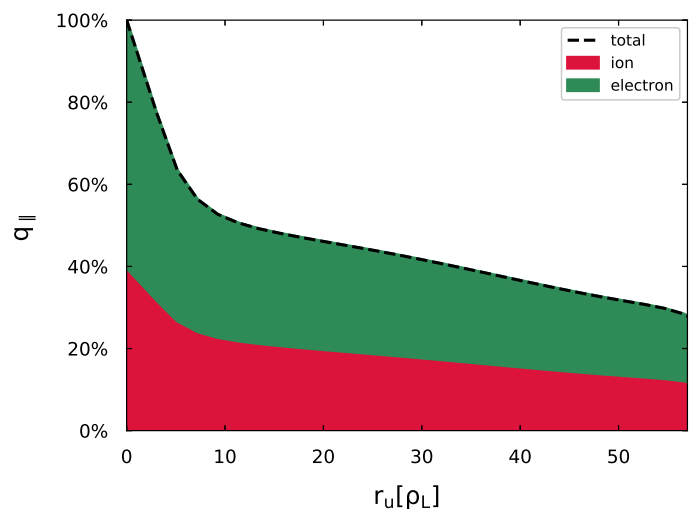

(c)

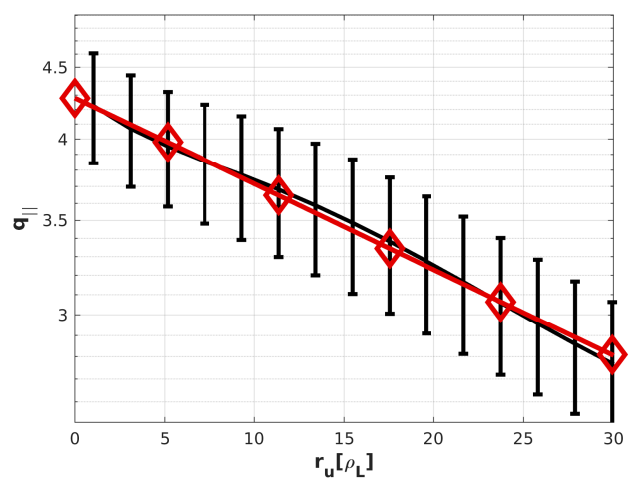

(b)

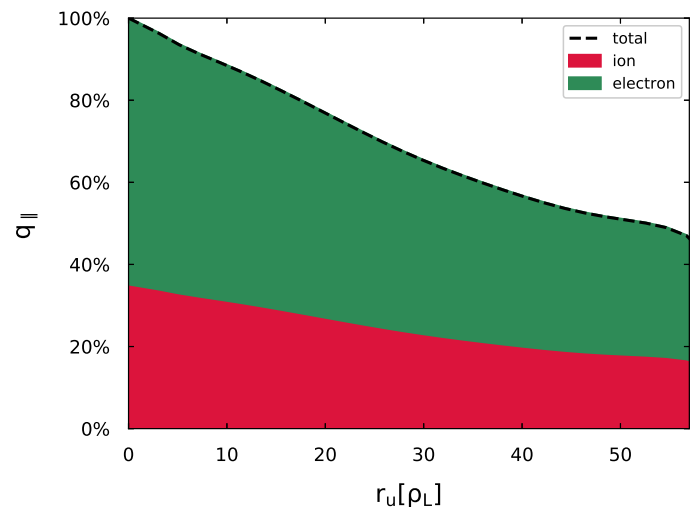

(d)

Figure 17: a-b) Magnification of profiles averaged in time and $\varphi$ direction of parallel heat into the near SOL at the LFS midplane for $\nu_{\star}=0.3 \nu_{\star, \text { ref }}$ (left) and $\nu_{\star}=10 \nu_{\star, \text { ref }}$ (right) showing the disappearance of the narrow feature when increasing collisionality. (c-d) Contribution of electrons and ions for the profiles (a, b). The vertical axis is normalized to the value of the $q_{\|}$ at the separatrix. Dashed black lines are the profiles of $q_{\|}$averaged in time and $\varphi$ direction into the whole SOL at the LFS midplane.

always dominant regardless of the collisionality. In the near SOL, they seem to be responsible for the formation and the disappearing of the narrow feature, although at $0.3 \nu_{\star, r e f}$ even the ions exhibit a smaller peak. Decreasing $\nu_{\star}$ appears to increase the ions' contribution in accordance, for example, with numerical studies performed in [84].

A simple estimate of the SOL width can be made balancing out the parallel $q_{\|} \simeq-\chi_{\|} \nabla_{\|} T_{e}$ and perpendicular $q_{\perp} \simeq-\chi_{\perp} \nabla_{\perp} T_{e}$ power:

$$
\frac{L_{\|}^{2}}{\chi_{\|}}=\frac{\lambda_{q}^{2}}{\chi_{\perp}}
$$

For the parallel thermal conductivity we can assume $\chi_{\|} \propto \nu_{\star}^{-1}$ (eq. (6) ). The dependence of temperature is neglected both because the value of $\chi_{e}$ is almost constant as shown in fig 2 and when $\nu_{\star}$ is small the temperature parallel gradient are almost null. The The estimate of $\chi_{\perp}$ could be made both from experimental [85, 86] or simulations data [87]. Generally we can assume that $\chi_{\perp}=a^{2} / \tau_{E}$ where $\tau_{E}$ is the energy confinement 
time. The scaling law provided in [88] by analysing experimental data of several machine during L-mode discharge it claims that the $\tau_{E}$ is proportional to $\nu_{\star}^{-0.19}$. Assuming this relation:

$$
\begin{aligned}
\lambda_{q} & =L_{\|} \sqrt{\frac{\chi_{\perp}}{\chi_{\|}}} \\
& \propto \sqrt{\frac{\nu_{\star}^{-0.19}}{\nu_{\star}^{-1}}} \propto \nu_{\star}^{0.4}
\end{aligned}
$$

Fitting the simulation data gives rise to a power law $\lambda_{q} \propto \nu_{\star}^{0.12}$. In fig 18 we display both scalings with their respective confidence interval. We realize that the diffusion model in (13) fails at $\nu_{\star} \leq 10 \nu_{\star}$ ref , whereas it seems to reproduce the trend for $\nu_{\star} \geq 100 \nu_{\star, r e f}$. A weak dependence of the $\lambda_{q}$ against $\nu_{\star}$ has been pointed out as well in thorough multi-machine experimental comparison [89] and in recent theoretical based on turbulent transport theory [90].It should be noted that $\lambda_{q}$ is strongly dependent from plasma parameters [91 95] which in turn are influenced by the variations of $\nu_{\star}$. This might reflect into changes of SOL width which are not taken into account in the simple estimate we made.

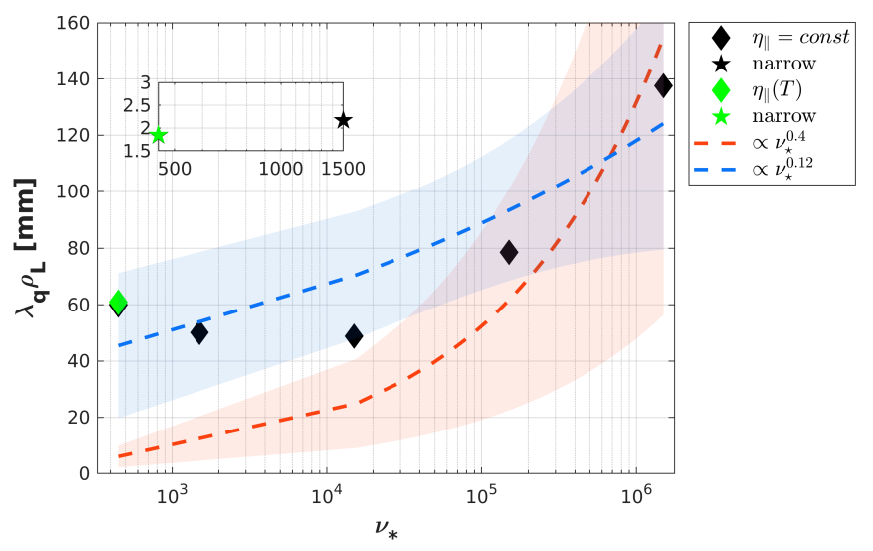

Figure 18: Variations of $\lambda_{q}$ with collisionality. $\lambda_{q}=\lambda_{q, f}$, (diamond) with a single exponential fit. $\lambda_{q, n}$ (stars) in the case where a double exponential fit is used. $\lambda_{q, f}$ and $\lambda_{q, n}$ are defined in Eq. 7. The case where the resistivity is temperature dependent overlaps with the lower collisionality case. The confidence interval at $95 \%$ is enlightened by the red area.

\subsection{Poloidal asymmetries}

The impact of collisionality on the parallel dynamics is now analyzed. Fig. 19 shows the poloidal distribution of the parallel Mach number, the radial turbulent particle flux (associated with density and $E \times B$ velocity fluctuations), and the radial convective and conductive heat fluxes.

The poloidal profiles of the Mach number are similar to those of the former isothermal studies [36]. They remain almost independent on the collisionality value 
and show a stagnation point close to the LFS midplane with a parallel flux becoming sonic at the HFS. When $\nu_{\star}>10 \nu_{\star}$,ref , close to the target plate a supersonic regime seems to be achieved $(M>2)$ due to the build-up of an effective particles sink [52]. No substantial change is shown concerning the particles and heat fluxes when changing $\nu_{\star}$ below $\nu_{\star, \text { ref }}$. They are poloidally concentrated at the outboard midplane, and the peak is slightly shifted towards the bottom of the machine. They decrease when approaching the top of the machine until a smaller peak is reached at the HFS on the targets. When $\nu_{\star}=10 \nu_{\star, \text { ref }}$ the ballooning character of radial fluxes becomes more marked. For higher collisionality values, the typical shape of the interchange ballooning disappears, the vast majority of convective fluxes seem to lie between the LFS midplane and the bottom of the machine. For these cases, large scale steady flows take over turbulence in radial transport and break the poloidal distribution.

Concerning the conductive heat flux, instead, it is rather homogeneously distributed over the whole poloidal domain, when $\nu_{\star} \leq 10 \nu_{\star}$ ref. A reduction of the heat conduction between the HFS and the LFS is observed when the $\nu_{\star}$ is raised up. This is due to the existence of larger poloidal asymmetries in temperature fluctuations, favored by a lower heat conductivity in these cases.
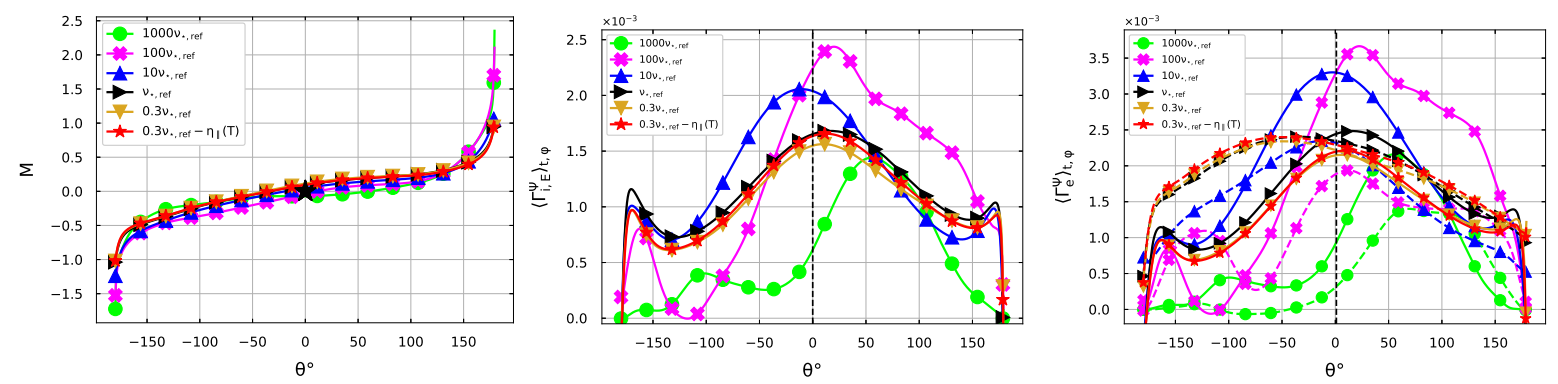

Figure 19: Poloidal distributions of the Mach number, the $\left\langle\tilde{N} \tilde{u}_{E}^{\Psi}\right\rangle$ component of radial particle flux, the heat radial flux at $r_{u}=5 \rho_{L}$ into the SOL. In latter figure solid lines represent the convective part and dashed lines represent the conductive part.

\subsection{Zonal Flows}

To investigate the impact of varying collisionality on zonal flows, we plot on Fig. 20 the spatio-temporal evolution of the particles flux in the $(r, t)$-plane for two collisionality values, $10 \nu_{\star, \text { ref }}$ and $0.3 \nu_{\star, \text { ref }}$. The long range quasi-coherent turbulence structures in the edge of the closed field lines region observed at $10 \nu_{\star, \text { ref }}$ are broken into smaller and less coherent structures at $0.3 \nu_{\star}$ ref . This can be related to a direct cascade of energy from the largest scales of the flow to the smallest ones.

It would be tempting to attribute this change in the pattern at low $\nu_{\star}$ to zonal flows. We have estimated the amplitude of zonal flows energy depending on the collisionality values. It is defined as the ratio between the energy of all fluctuating 

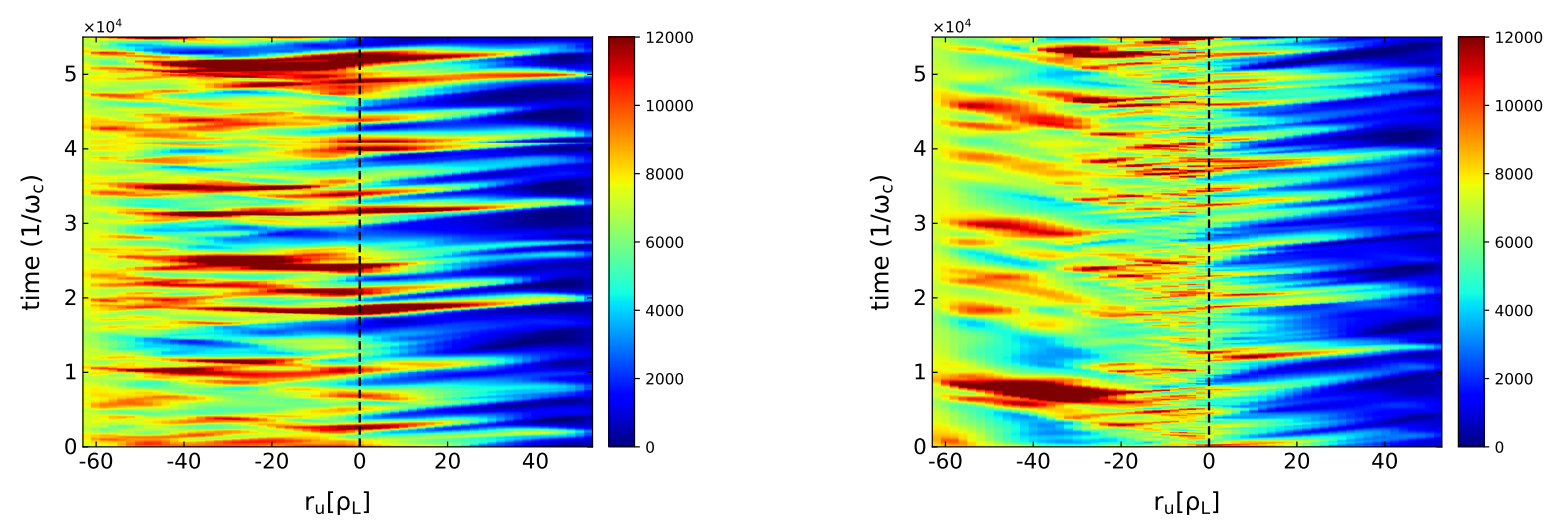

Figure 20: 2D spatio-temporal evolutions of the particles flux averaged on the flux surfaces in the $(r, t)$ plane at $10 \nu_{\star, \text { ref }}$ (left) and at $0.3 \nu_{\star, \text { ref }}$ (right). The black line denotes the separatrix.

$\left(\omega \neq 0, k_{\theta}=0, k_{\varphi}=0\right)$-modes of $v_{E, \theta}$ and the energy of all modes as:

$$
E_{Z F}=\frac{\sum_{\omega} \tilde{v}_{E, \theta}^{2}\left(\omega \neq 0, r, k_{\theta}=0, k_{\varphi}=0\right)}{\sum_{\omega, k_{\theta}, k_{\varphi}} \tilde{v}_{E, \theta}^{2}\left(\omega \neq 0, r, k_{\theta}, k_{\varphi}\right)}
$$

which is plotted on Fig. 21. Results show that the relative energy of zonal flow increases when decreasing the collisionality value. Meanwhile, the fluctuations level decreases as shown in Fig. 10. Therefore, these results suggest that a part of the turbulent energy is transferred from the small turbulence scales to the zonal flow when decreasing the collisionality [96]. This behavior would suggest an inverse cascade mechanism that could partially explain the decrease of the turbulent flux.

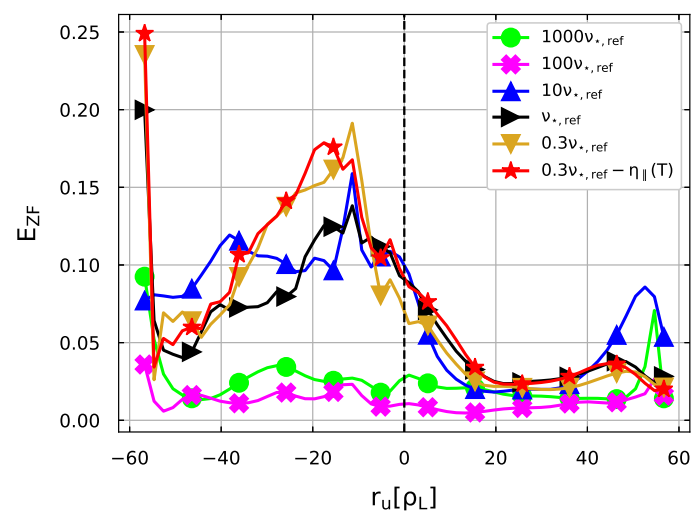

Figure 21: Radial profile of the relative energy of the zonal flow with respect to the total energy $E_{Z F}$ (Eq. 14 averaged in time and $\varphi$ direction and depending on the collisionality.

\section{Concluding remarks}

The present study based on TOKAM3X non-isothermal fluid turbulence simulations has investigated the impact of changing the plasma collisionality $\nu_{\star}$ on plasma properties in 
the tokamak edge. The study has been performed in a circular limited L-mode plasma with the limiter placed at the high field side (HFS) midplane. In the considered model, changing $\nu_{\star}$ impacts self-consistently the parallel resistivity and the parallel thermal conductivities of the ions and the electrons.

We have first reported results for a reference simulation at $\nu_{\star}=\nu_{\star r e f}=1500$ corresponding to realistic conditions for the COMPASS tokamak. The flow properties are in general agreement with former isothermal studies and experimental observations. The interchange dominated turbulence is ballooned at the LFS, with an intermittency behavior dominated by small-amplitude events in the core-edge region (nearly Gaussian PDFs) and characterized by the increasing appearance of large-amplitude events into the SOL (positively skewed PDFs). The SOL radial profiles of parallel heat flux exhibit a double scale length, where it appears the so-called narrow feature. Besides, results show that the heat conduction and convection generated by turbulence fluctuations carry most of the radial energy flux. While convection dominates both perpendicular particle and heat fluxes in the edge-core region, a significant increment of the conductive heat flux is observed in the SOL.

Simulations with four different collisionality values have been performed to compare against the reference case with $\nu_{\star} \in\left[0.3 \nu_{\star, r e f}, 1000 \nu_{\star, r e f}\right]$. A supplementary simulation at $0.3 \nu_{\star, \text { ref }}$ has also been run, including the temperature dependency of the parallel resistivity. In the latter case, the radial distribution of $\eta_{\|}$shows the presence of two zones of almost constant resistivity in the core-edge region and SOL with a rapid transition around the separatrix of an order of magnitude. However, our analyses do not show any significant variations with respect to the simulation performed at the same collisionality with a spatially constant parallel resistivity $\eta_{\|}=\eta_{\| 0}$.

Present results allow us to draw the following main conclusions:

- Changing collisionality has a stronger impact in the edge of the closed field lines region rather than in the SOL. This is related to the fact that charge circulation in the SOL is governed by the sheath resistance which dominates plasma resistivity below a certain collisionality value.

- In the closed field lines region, the general trend is a stabilization of interchange driven turbulence at low collisionality leading to a reduction of the fluctuations level and an enlargement of the gradient of the equilibrium profiles. The increase of the radial gradients is especially marked for temperatures in the vicinity of the separatrix. The steepening of gradients is followed by an increase of the shear of the radial electric field at the separatrix and has a local impact on the radial transport. Nevertheless, it does not explain the global reduction of turbulent transport in the core-edge region. These results suggest, however, that lowering collisionality strengthens the energy level of zonal flow. It was found that collisionality influences zonal flow dynamics as predicted by gyro-kinetic theory. The interplay between the 
turbulent transport and zonal flow could lead to destroy the coherence of avalanche driving transport events.

- The $E \times B$ driven turbulent transport of particles and the convective heat transport are highly reduced at low collisionality values, whereas the diffusive particles and fluctuations driven conductive heat transport are enhanced

- The scaling of the SOL width $\lambda_{q}$ is weakly sensitive on the collisionality values with resulting power dependence equal to $\simeq 0.12$. The results show two different transport dynamics in the closed field lines region and in the SOL that could explain the formation of narrow feature. For collisionality value low enough, the transition between the two zones is not instantaneous, but it is ruled by the presence of the layer at the beginning of the SOL that is a reminiscence of the edge-core transport. Lowering the collisionality value strengthens the existence of the narrow feature. Moreover, associated to the presence of the narrow feature, a higher level of conductive heat transport is observed in accordance with experimental observation.

- The formation of density shoulder solely related to an increasing collisionality seems to be incompatible with our results when $\nu_{\star} \leq 10 \nu_{\star, r e f}$.

\section{Acknowledgement}

This work has been carried out within the framework of the EUROfusion Consortium and has received funding from the Euratom research and training programme 2014-2018 and 2019-2020 under grant agreement No 633053. The views and opinions expressed herein do not necessarily reflect those of the European Commission. This work has been carried out thanks to the support of the A*MIDEX project (ANR-11-IDEX-0001 02, TOP project) funded by the 'Investissements d'Avenir' French Government program, managed by the French National Research Agency (ANR). This work was granted access to the HPC resources of IDRIS under the allocations A0030506912 \& A0050506912 made by GENCI, of Aix-Marseille University, financed by the project Equip@Meso (ANR-10EQPX-29 01), and of the EUROfusion High Performance Computer (Marconi-Fusion) under the project HEAT.

\section{Appendix A. The TOKAM3X equations}

The TOKAM3X code solves 3D drift-reduced Braginskii fluid equations for the study of edge plasma turbulence [97]. It accounts for the dynamic of electrons and ions dynamic, and only fluctuations of the electric potential are considered (electrostatic assumption). The isothermal model detailed in [33] is extended here to non-isothermal plasmas, including energy balance equations.

The magnetic field is fixed and axisymmetric. The vector $\vec{b}=\vec{B} / B$ defines the direction of the magnetic field. The gradients in the parallel and perpendicular directions are respectively defined as $\nabla_{\|} \cdot=\vec{b} \cdot \nabla \cdot$ and $\nabla_{\perp} \cdot=\nabla \cdot-\vec{b} \nabla_{\|} \cdot$ 
The first-order electric and curvature drift velocities $u_{\perp e i}$ are defined such that:

$$
\begin{aligned}
& u_{\perp e}=u_{E}+u_{\nabla B e}=\frac{B \times \nabla \Phi}{B^{2}}-\frac{2 T_{e}}{B} \frac{B \times \nabla B}{B^{2}} \\
& u_{\perp i}=u_{E}+u_{\nabla B i}=\frac{B \times \nabla \Phi}{B^{2}}+\frac{2 T_{i}}{B} \frac{B \times \nabla B}{B^{2}}
\end{aligned}
$$

The curvature drift $B \times \nabla B$ replaces the diamagnetic drift $\left(u_{\text {diam }}=(B \times \nabla P) / B^{2}\right)$ to explicitly account for the so called "diamagnetic cancellation", [98] [99].

The dimensionless equations (reference scales in Tab.1) for the electronic density $N$, the ionic parallel momentum $\Gamma$, the electrostatic potential $\Phi$, the energy for the electrons and the ions, and the parallel current $j_{\|}$which defines the parallel advection velocity for electrons are the following:

$$
\begin{aligned}
& \partial_{t} N+\nabla \cdot\left[N\left(u_{\| i} \vec{b}+N \vec{u}_{\perp i}\right)\right]=\nabla \cdot\left(D_{\perp N} \nabla N\right)+S_{N} \\
& \partial_{t} \Gamma_{\|, i}+\nabla \cdot\left[\Gamma_{\|, i}\left(u_{\| i} \vec{b}+\vec{u}_{\perp i}\right)\right]=-\nabla_{\|}\left(P_{i}+P_{e}\right) \\
& +\nabla \cdot\left(D_{\perp \Gamma} N \nabla\left(u_{\| i}\right)\right)+\nabla \cdot\left(D_{\perp N} u_{\| i} \nabla N\right)+S_{\Gamma_{\|, i}} \\
& \partial_{t}\left(\frac{3}{2} P_{e}\right)+\nabla \cdot\left[\frac{5}{2} P_{e}\left(u_{\| i} \vec{b}+\vec{u}_{\perp e}\right)\right]+\nabla \cdot \vec{q}_{e}= \\
& +u_{\perp e} \cdot \nabla P_{e}-u_{\| i} \vec{b} \cdot \nabla P_{e}+Q_{\| e} \\
& +\nabla \cdot\left(D_{\perp T_{e}} N \nabla_{\perp} T_{e}\right)+\nabla \cdot\left(D_{\perp N} \frac{3}{2} T_{e} \nabla_{\perp} N\right)+S_{E e} \\
& \partial_{t}\left(\frac{3}{2} P_{i}+\frac{1}{2} N u_{\| i}^{2}\right)+\nabla \cdot\left[\left(\frac{5}{2} P_{i}+\frac{1}{2} N u_{\| i}^{2}\right)\left(\vec{u}_{\perp i}+u_{\| i} \vec{b}\right)\right]+\vec{\nabla} \cdot \vec{q}_{i}= \\
& +u_{\perp i} \cdot \nabla P_{i}+u_{\| i} \vec{b} \cdot \nabla P_{e}+Q_{\| i}+\nabla \cdot\left(D_{\perp T_{i}} N \nabla_{\perp} T_{i}\right) \\
& \nabla \cdot\left[D_{\perp N}\left(\frac{3}{2} T_{i}+\frac{1}{2} u_{\| i}^{2}\right) \nabla_{\perp} N\right]+\nabla \cdot\left(D_{\perp \Gamma} \Gamma_{\|, i} \nabla u_{\| i}\right)+S_{E i} \\
& N \nabla_{\|} \Phi=\nabla_{\|} P_{e}+0.71 N \nabla_{\|} T_{e}-\eta_{\|} N j_{\|} \\
& \partial_{t} W+\vec{\nabla} \cdot\left(W u_{\| i} \vec{b}+W \vec{u}_{E}+W \vec{u}_{\nabla B i}\right) \\
& =\vec{\nabla} \cdot\left(N\left(\vec{u}_{\nabla B i}-\vec{u}_{\nabla B e}\right)\right)+\vec{\nabla} \cdot\left(J_{\|} \vec{b}\right) \\
& +\vec{\nabla} \cdot\left(D_{\perp W} \vec{\nabla} W\right)+S_{W}
\end{aligned}
$$

Equation (A.7) corresponds to the charge conservation $(\nabla \cdot J=0)$ equation and the equation A.6 is the generalized Ohm's law.

$W=\nabla \cdot \frac{1}{B^{2}}\left(\nabla_{\perp} \Phi+\nabla_{\perp} T_{i}+T_{i} \nabla_{\perp} \log N\right)$ defines the vorticity under the Boussinesqlike approximation [100], $p_{e, i}=N T_{e, i}$ is the pressure, $Q_{\| e / i}= \pm 3 \frac{m_{e}}{m_{i}} \nu_{\star}^{T K 3 X} \frac{N}{\tau_{e}}\left(T_{e}-T_{i}\right)$ 
is the collisional the energy equipartion term between ions and electrons. $\eta_{\|} N j_{\|}-$ $0.71 N \nabla_{\|} T_{e}=R_{\|}$is a friction force due to collisions. $q_{e / i}=-\chi_{\| e / i} \nabla_{\|} T_{e / i} \vec{b}$ is the conductive term in the electron and ion energy equations where $\chi_{\| e / i}$ are the thermal conductivties for electrons and ions. The perpendicular diffusion terms account for other transport mechanisms not included in the model such as collision driven classical and neoclassical transport. They are also needed to damp fluctuations of scales smaller than the grid size [33].

\section{Appendix A.1. Resistivity in TOKAM3X}

This section describes the procedure to get the dimensionless parallel resistivity $\eta_{\|}$used in TOKAM3X. The starting point is the expression derived by Braginskii: $($ )

$$
\eta_{\|}^{B}=\frac{0.51 m_{e}}{n_{e} e^{2} \tau_{e i}}
$$

where $n_{e}$ and $\tau_{e i}$ are the dimensional density and electron-ion collision time measured respectively in $m^{-3}$ and seconds $s$, and $\eta_{\|}^{B}$ is evaluated in $\Omega m . \tau_{e i}$ is defined as:

$$
\tau_{e i}=\frac{3(2 \pi)^{3 / 2} \epsilon_{0}^{2} \sqrt{m_{e}} \hat{T}_{e}^{3 / 2}}{n_{e} e^{5 / 2} \ln \Lambda}
$$

here $\hat{T}_{e}$ is the electron temperature in $e V$ and $\ln \Lambda$ is the Coulomb logarithm which is equal to $5.2-0.5 \ln \left(n_{e} / 10^{20}\right)+\ln \hat{T}_{e}$ for $\hat{T}_{e} \geq 10 \mathrm{eV}$. Therefore the adimensional resistivity $\eta_{\|}$is given by the ratio between $\eta_{\|}^{B}$ and the reference resitivity $\eta_{0}$, equal to $B_{0} / e n_{0}$ (see Tab. 1):

$$
\eta_{\|}=0.51 \frac{m_{e}}{e B_{0}} \frac{n_{0}}{n_{e} \tau_{e i}}=0.51 \frac{m_{e}}{m_{i}} \frac{1}{\Omega_{i}^{0} N \tau_{e i}}
$$

here $N=n_{e} / n_{0}$ and $\Omega_{i}^{0}=e B_{0} / m_{i}$ are the dimensionless density and the reference ion cyclotron frequency. Since both $\tau_{e i}$ and $\ln \Lambda$ depend on the density and temperature, it is convenient to explicit the dependence in terms of the adimensional density $N$ and temperature $T_{e}$. Hence we can rewrite Eq. A.9 as it follows:

$$
\tau_{e i}=\frac{3(2 \pi)^{3 / 2} \epsilon_{0}^{2} \sqrt{m_{e}} T_{0}^{3 / 2}}{e^{5 / 2} n_{0} \ln \Lambda_{0}} \frac{\hat{T}_{e}^{3 / 2} n_{0}}{n_{e} T_{0}^{3 / 2}} \frac{\ln \Lambda_{0}}{\ln \Lambda}=\tau_{e i}^{r e f} \frac{T_{e}^{3 / 2}}{N} \ln \Lambda^{\star}
$$

where $\ln \Lambda_{0}$ is the Coulomb logarithm calculated using the reference parameters in Tab. 1 and $\ln \Lambda^{\star}$ is the ratio $\ln \Lambda_{0} / \ln \Lambda$. As shown in the Wesson Appendix, the Coulomb logarithm varies between $13-20$ over a wide range of temperature and density values. Therefore, it is safe to assume that $\Lambda^{\star} \simeq 1$. Then, we can substitute Eq.A.11 into Eq.A.10 getting to:

$$
\eta_{\|}=0.51 \frac{m_{e}}{m_{i}} \frac{1}{N} \frac{N}{T_{e}^{3 / 2}} \frac{1}{\Omega_{i}^{0} \tau_{e i}^{r e f}}
$$

$\ddagger$ Reference [99] contains all relations used in this paragraph 
$\tau_{e i}^{r e f}$ being the ion collision time calculated with reference parameters in Tab. 1. The term $\left(\Omega_{i}^{0} \tau_{e i}^{r e f}\right)^{-1}$ can be written as $\rho_{\star} \nu_{\star}^{\prime}$, where $\rho_{\star}=\rho_{L} / a$ and $\nu_{\star}^{\prime}=a /\left(c_{s}^{0} \tau_{e i}^{r e f}\right)$ leading to the definition of collisionality used into TOKAM3X defined:

$$
\nu_{\star}^{T K 3 X}=\rho_{\star} \nu_{\star}^{\prime}=\frac{\rho_{L}}{a} \frac{a}{c_{s} \tau_{e i}^{r e f}}=\frac{1}{\Omega_{i}^{0} \tau_{e i}^{r e f}}
$$

Comparing the latter expression with the definition of $\nu_{\star}$ given in Eq. (4) we get the following equivalence:

$$
\begin{aligned}
\nu_{\star} & =\frac{2 \pi q \mathcal{A}}{\rho_{\star}} \nu_{\star}^{T K 3 X} \\
\eta_{\|} & =0.51 \frac{m_{e}}{m_{i}} \frac{\rho_{\star}}{2 q \pi \mathcal{A}} \nu_{\star} T_{e}^{-\frac{3}{2}}
\end{aligned}
$$

where we recover the definition of the parallel resistivity given in (6). The same procedure can be applied to $\chi_{\| e, i}$ knowing that the dimensional parallel ionic and electronic thermal conductivities are given by $\chi_{\|, e}=3.16 \frac{p_{e} \tau_{e i}}{m_{e}}$ and $\chi_{\|, i}=3.9 \frac{p_{i} \tau_{i}}{m_{i}}$, where $\tau_{i}$ is the ion collision time which can be written as function of $\tau_{e i}: \tau_{i} \simeq\left(\frac{m_{i}}{m_{e}}\right)^{1 / 2} \tau_{e i}$.

\section{Appendix B. Sheath resistivity}

In this section we derive a relation between the parallel and sheath resistance. In TOKAM3X model the sheath is not treated explicitly into the code, but we account for its presence through the Bohm boundary condition (Eq. (1)). The procedure has carried out starting from dimensional quantity. If we linearize this condition we can get a ratio between a potential the sheath and the current entering into the sheath that we define as $R_{\text {sheath }}$

$$
\begin{aligned}
& j_{\|}=\frac{j_{\text {sat }} e}{\hat{T}_{e}}\left(\frac{\Lambda \hat{T}_{e}}{e}-\Phi\right) \\
& R_{\text {sheath }}=\frac{\frac{\Lambda \hat{T}_{e}}{e}-\Phi}{j_{\|}}=\frac{\hat{T}_{e}}{e j_{\text {sat }}}
\end{aligned}
$$

The parallel resistance of the plasma is taken using (A.8):

$$
R_{\|}=\eta_{\|} L_{\|}=0.51 \frac{m_{e} \nu_{e i} L_{\|}}{e^{2} n_{e}}
$$


Therefore the ratio between

$$
\begin{aligned}
\frac{R_{\text {sheath }}}{R_{\|}} & =\frac{\hat{T}_{e} e^{2} n_{e}}{0.51 e m_{e} \nu_{e i} L_{\|} j_{s a t}}=\frac{\hat{T}_{e}}{0.51 m_{e} \nu_{e i} L_{\|} \hat{c}_{s}}= \\
& =\frac{\hat{T}_{e} c_{s}^{0}}{0.51 m_{e} \nu_{e i}^{0} \frac{N}{T_{e}^{3}} L_{\|} \hat{c}_{s} c_{s}^{0}}=\sqrt{\frac{\hat{T}_{e}^{2}}{\frac{m_{e}^{2}}{m_{i}^{2}}\left(\hat{T}_{e}+\hat{T}_{i}\right) T_{0}}} \frac{1}{0.51 \nu_{\star}} \frac{T_{e}^{3 / 2}}{N} \\
& \stackrel{T_{e} \simeq T_{i}}{\longrightarrow} \simeq \frac{m_{i}}{2 m_{e}} \frac{T_{e}^{1 / 2}}{0.51 \nu_{\star}} \frac{T_{e}^{3 / 2}}{N}=\frac{m_{i}}{m_{e}} \frac{1}{\nu_{\star}} \frac{T_{e}^{2}}{N} \simeq 2000 \frac{1}{\nu_{\star}} \frac{T_{e}^{2}}{N}
\end{aligned}
$$

Here we have considered $\hat{c}_{s}$ as the dimensional ion sound speed equal to $\sqrt{\left(\hat{T}_{e}+\hat{T}_{i}\right) / m_{i}}$. We find that $R_{\text {sheath }} / R_{\|}>>1$, when $\nu_{\star}<<2000 T_{e}^{2} / N$. Assuming that $T_{e}^{2} / N \simeq$ 1 ,meaning being on separatrix, the relation become $\nu_{\star}<<2000$. Therefore in the case $\nu_{\star} \leq \nu_{\star, \text { ref }}$ the resistance of the sheath rule the SOL dynamics. We mention as well that if we consider a deuterium plasma the mass ration will be doubled enhancing the strength of the shear. A similar relation has been found in [101].

\section{References}

[1] Stangeby P C 2000 The plasma boundary of magnetic fusion devices (CRC Press)

[2] Wootton A, Carreras B, Matsumoto H, McGuire K, Peebles W, Ritz C P, Terry P and Zweben S 1990 Physics of Fluids B: Plasma Physics 2 2879-2903

[3] Liewer P C 1985 Nuclear Fusion 25543

[4] Ritz C P, Bravenec R, Schoch P, Bengtson R D, Boedo J, Forster J, Gentle K W, He Y, Hickok R, Kim Y et al. 1989 Physical review letters 621844

[5] Zweben S, Boedo J, Grulke O, Hidalgo C, LaBombard B, Maqueda R, Scarin P and Terry J 2007 Plasma Physics and Controlled Fusion 49 S1

[6] Rudakov D, Boedo J, Moyer R, Stangeby P C, Watkins J, Whyte D, Zeng L, Brooks N, Doerner R, Evans T et al. 2005 Nuclear fusion 451589

[7] LaBombard B, Boivin R, Greenwald M, Hughes J, Lipschultz B, Mossessian D, Pitcher C, Terry J, Zweben S and Group A 2001 Physics of Plasmas 8 2107-2117

[8] Lipschultz B, Whyte D and LaBombard B 2005 Plasma physics and controlled fusion $\mathbf{4 7} 1559$

[9] Myra J, Russell D and D'Ippolito D 2006 Physics of plasmas 13112502

[10] Garcia O E, Pitts R, Horacek J, Madsen J, Naulin V, Nielsen A H and Rasmussen J J 2007 Plasma Physics and Controlled Fusion 49 B47

[11] Theiler C, Furno I, Ricci P, Fasoli A, Labit B, Müller S and Plyushchev G 2009 Physical review letters 103065001

[12] Carralero D, Birkenmeier G, Müller H, Manz P, DeMarne P, Müller S, Reimold F, Stroth U, Wischmeier M, Wolfrum E et al. 2014 Nuclear Fusion 54123005 
[13] Wynn A, Lipschultz B, Cziegler I, Harrison J, Jaervinen A, Matthews G, Schmitz J, Tal B, Brix M, Guillemaut C et al. 2018 Nuclear Fusion 58056001

[14] Vianello N, Tsui C, Theiler C, Allan S, Boedo J, Labit B, Reimerdes H, Verhaegh K, Vijvers W, Walkden N et al. 2017 Nuclear Fusion 57116014

[15] Carralero D, Manz P, Aho-Mantila L, Birkenmeier G, Brix M, Groth M, Müller H, Stroth U, Vianello N, Wolfrum E et al. 2015 Physical review letters 115215002

[16] Carralero D, Müller H, Groth M, Komm M, Adamek J, Birkenmeier G, Brix M, Janky F, Hacek P, Marsen S et al. 2015 Journal of Nuclear Materials 463 123-127

[17] Scott B D 2005 Physics of Plasmas 12062314

[18] Mosetto A, Halpern F D, Jolliet S, Loizu J and Ricci P 2013 Physics of Plasmas 20092308

[19] Mosetto A, Halpern F D, Jolliet S and Ricci P 2012 Physics of Plasmas 19112103

[20] Rogers B, Drake J and Zeiler A 1998 Physical Review Letters 814396

[21] Osborne T, Groebner R, Smith S, Snyder P, Thomas D and Yan Z 2013 Effect of collisionality on h-mode pedestal structure APS Meeting Abstracts

[22] Rosenbluth M and Hinton F 1998 Physical review letters 80724

[23] Smolyakov A, Diamond P and Malkov M 2000 Physical review letters 84491

[24] Diamond P H, Itoh S, Itoh K and Hahm T 2005 Plasma Physics and Controlled Fusion 47 R35

[25] Lin Z, Hahm T, Lee W, Tang W and Diamond P 1999 Physical review letters 83 3645

[26] Falchetto G and Ottaviani M 2004 Physical review letters 92025002

[27] Norscini C 2015 Self-organized turbulent transport in fusion plasmas Ph.D. thesis Aix Marseille Universite

[28] Ghendrih P, Asahi Y, Caschera E, Dif-Pradalier G, Donnel P, Garbet X, Gillot C, Grandgirard V, Latu G, Sarazin Y et al. 2018 Generation and dynamics of sol corrugated profiles Journal of Physics: Conference Series vol 1125 (IOP Publishing) p 012011

[29] Zeiler A, Drake J and Rogers B 1997 Physics of Plasmas 4 2134-2138

[30] Stegmeir A, Coster D, Ross A, Maj O, Lackner K and Poli E 2018 Plasma Physics and Controlled Fusion 60035005

[31] Ricci P, Halpern F, Jolliet S, Loizu J, Mosetto A, Fasoli A, Furno I and Theiler C 2012 Plasma Physics and Controlled Fusion 54124047

[32] Dudson B D, Allen A, Breyiannis G, Brugger E, Buchanan J, Easy L, Farley S, Joseph I, Kim M, McGann A et al. 2014 arXiv preprint arXiv:1405.7905

[33] Tamain P, Bufferand H, Ciraolo G, Colin C, Galassi D, Ghendrih P, Schwander F and Serre E 2016 Journal of Computational Physics 321 606-623

[34] Tamain P, Bufferand H, Ciraolo G, Colin C, Ghendrih P, Schwander F and Serre E 2014 Contributions to Plasma Physics 54 555-559 
[35] Tamain P, Ghendrih P, Bufferand H, Ciraolo G, Colin C, Fedorczak N, Nace N, Schwander F and Serre E 2015 Plasma Physics and Controlled Fusion 57054014

[36] Galassi D, Tamain P, Bufferand H, Ciraolo G, Ghendrih P, Baudoin C, Colin C, Fedorczak N, Nace N and Serre E 2017 Nuclear Fusion 57036029

[37] Gallo A, Fedorczak N, Elmore S, Maurizio R, Reimerdes H, Theiler C, Tsui C, Boedo J, Faitsch M, Bufferand $\mathrm{H}$ et al. 2017 Plasma Physics and Controlled Fusion 60014007

[38] Nespoli F, Tamain P, Fedorczak N, Galassi D and Marandet Y 2020 Nuclear Fusion 60046002

[39] Fan D, Marandet Y, Tamain P, Bufferand H, Ciraolo G, Ghendrih P and Serre E 2019 Nuclear Materials and Energy 18 105-110

[40] Cartier-Michaud T, Galassi D, Ghendrih P, Tamain P, Schwander F and Serre E 2020 Accepted for publication, Physics of Plasmas Conference series

[41] Galassi D 2017 Numerical modelling of transport and turbulence in tokamak edge plasma with divertor configuration Ph.D. thesis Aix-Marseille

[42] Madsen J, Naulin V, Nielsen A H and Rasmussen J J 2016 Physics of Plasmas 23032306

[43] Krasheninnikov S, D'ippolito D and Myra J 2008 Journal of Plasma Physics 74 679-717

[44] Boedo J, Rudakov D, Colchin R, Moyer R, Krasheninnikov S, Whyte D, McKee G, Porter G, Schaffer M, Stangeby P et al. 2003 Journal of nuclear materials 313 813-819

[45] Silva C, Goncalves B, Hidalgo C, Pedrosa M, Erents K, Matthews G and Pitts R 2004 Review of scientific instruments $\mathbf{7 5}$ 4314-4316

[46] Adamek J, Horacek J, Müller H, Rohde V, Ionita C, Schrittwieser R, Mehlmann F, Kurzan B, Stöckel J, Dejarnac R et al. 2010 Contributions to Plasma Physics $50854-859$

[47] Allan S, Elmore S, Fishpool G, Dudson B, Team M, Team E M et al. 2016 Plasma Physics and Controlled Fusion 58045014

[48] Ryter F, Orte L B, Kurzan B, McDermott R, Tardini G, Viezzer E, Bernert M, Fischer R et al. 2014 Nuclear Fusion 54083003

[49] LaBombard B, Rice J, Hubbard A, Hughes J, Greenwald M, Irby J, Lin Y, Lipschultz B, Marmar E, Pitcher C et al. 2004 Nuclear fusion 441047

[50] Gunn J, Boucher C, Dionne M, Duran I, Fuchs V, Loarer T, Nanobashvili I, Pánek R, Pascal J Y, Saint-Laurent F et al. 2007 Journal of Nuclear Materials $363484-490$

[51] Asakura N, SOL I and Group D T 2007 Journal of nuclear materials 363 41-51

[52] Ghendrih P, Bodi K, Bufferand H, Chiavassa G, Ciraolo G, Fedorczak N, Isoardi L, Paredes A, Sarazin Y, Serre E et al. 2011 Plasma Physics and Controlled Fusion 53054019 
[53] Colin C, Tamain P, Schwander F, Serre E, Bufferand H, Ciraolo G, Fedorczak N and Ghendrih P 2015 Journal of Nuclear Materials 463 654-658

[54] Nespoli F, Labit B, Furno I, Horacek J, Tsui C, Boedo J, Maurizio R, Reimerdes H, Theiler C, Ricci P et al. 2017 Nuclear Fusion 57126029

[55] Horacek J, Vondracek P, Panek R, Dejarnac R, Komm M, Pitts R, Kocan M, Goldston R J, Stangeby P, Gauthier E et al. 2015 Journal of Nuclear Materials 463 385-388

[56] Kocan M, Pitts R, Arnoux G, Balboa I, De Vries P, Dejarnac R, Furno I, Goldston R J, Gribov Y, Horacek J et al. 2015 Nuclear Fusion 55033019

[57] Naulin V 2007 Journal of nuclear materials 363 24-31

[58] Carreras B, Hidalgo C, Sánchez E, Pedrosa M, Balbin R, García-Cortés I, Van Milligen B, Newman D and Lynch V 1996 Physics of Plasmas 3 2664-2672

[59] Galassi D, Ciraolo G, Tamain P, Bufferand H, Ghendrih P, Nace N and Serre E 2019 Fluids 450

[60] Liewer P, McChesney J, Zweben S and Gould R 1986 The Physics of fluids 29 309-317

[61] Boedo J, Terry P, Gray D, Ivanov R, Conn R, Jachmich S, Van Oost G, Team T et al. 2000 Physical review letters $\mathbf{8 4} 2630$

[62] Meier M A, Bengtson R D, Hallock G A and Wootton A J 2001 Physical review letters 87085003

[63] Garcia O, Horacek J and Pitts R 2015 Nuclear Fusion 55062002

[64] Berk H, Ryutov D and Tsidulko Y A 1991 Physics of Fluids B: Plasma Physics 3 1346-1354

[65] Sarazin Y, Ghendrih P, Attuel G, Clément C, Garbet X, Grandgirard V, Ottaviani M, Benkadda S, Beyer P, Bian N et al. 2003 Journal of nuclear materials 313 796803

[66] Manz P, Ribeiro T, Scott B, Birkenmeier G, Carralero D, Fuchert G, Müller S, Müller H, Stroth U and Wolfrum E 2015 Physics of Plasmas 22022308

[67] Ryutov D and Cohen R 2004 Contributions to plasma physics 44 168-175

[68] Farina D, Pozzoli R and Ryutov D 1993 Plasma physics and controlled fusion 35 1271

[69] Vianello N, Tsui C, Theiler C, Allan S, Boedo J, Labit B, Reimerdes H, Verhaegh K, Vijvers W, Walkden N et al. 2016 On filamentary transport in the tcv tokamak: Addressing the role of the parallel connection length Proc. 26th IAEA Fusion Energy Conf

[70] Easy L, Militello F, Omotani J, Walkden N and Dudson B 2016 Physics of Plasmas 23012512

[71] Ribeiro T and Scott B 2008 Plasma Physics and Controlled Fusion 50055007 
[72] Terry J, Zweben S, Hallatschek K, LaBombard B, Maqueda R, Bai B, Boswell C, Greenwald M, Kopon D, Nevins W et al. 2003 Physics of Plasmas 10 1739-1747

[73] LaBombard B, Umansky M, Boivin R, Goetz J, Hughes J, Lipschultz B, Mossessian D, Pitcher C, Terry J et al. 2000 Nuclear Fusion 402041

[74] Militello F, Garzotti L, Harrison J, Omotani J, Scannell R, Allan S, Kirk A, Lupelli I, Thornton A et al. 2015 Nuclear Fusion 56016006

[75] Asakura N, Hosogane N, Itami K, Sakasai A, Sakurai S, Shimizu K, Shimada M, Kubo H, Higashijma S, Takenaga H et al. 1999 Journal of nuclear materials 266 $182-188$

[76] Militello F and Omotani J 2016 Nuclear Fusion 56104004

[77] Burrell K 2020 Physics of Plasmas 27060501

[78] Biglari H, Diamond P and Terry P 1990 Physics of Fluids B: Plasma Physics 2 $1-4$

[79] Waltz R, Dewar R and Garbet X 1998 Physics of Plasmas 5 1784-1792

[80] Loizu J, Ricci P, Halpern F, Jolliet S and Mosetto A 2013 Plasma Physics and Controlled Fusion 55124019

[81] Garbet X, Fenzi C, Capes H, Devynck P and Antar G 1999 Physics of Plasmas 6 3955-3965

[82] Jorge R, Ricci P, Halpern F D, Loureiro N F and Silva C 2016 Physics of Plasmas 23102511

[83] Halpern F D and Ricci P 2016 Nuclear Fusion 57034001

[84] Nielsen A H, Rasmussen J J, Madsen J, Xu G, Naulin V, Olsen J M B, Løiten M, Hansen S, Yan N, Tophøj L et al. 2016 Plasma Physics and Controlled Fusion 59 025012

[85] Loarte A, Bosch S, Chankin A, Clement S, Herrmann A, Hill D, Itami K, Lingertat J, Lipschultz B, McCormick K et al. 1999 Journal of nuclear materials 266 587592

[86] Silvagni D, Eich T, Faitsch M, Happel T, Sieglin B, David P, Nille D, Gil L, Stroth U et al. 2020 Plasma Physics and Controlled Fusion 62045015

[87] Caschera E 2019 Global confinement properties of Tokamak plasmas in global, flux-driven, gyrokinetic simulations Ph.D. thesis Aix Marseille Universite

[88] ITER P E G o C, Trasport, Modelling C and Database 1999 Nuclear Fusion 39

[89] Horacek J, Pitts R, Adamek J, Arnoux G, Bak J, Brezinsek S, Dimitrova M, Goldston R J, Gunn J, Havlicek J et al. 2016 Plasma Physics and Controlled Fusion $\mathbf{5 8} 074005$

[90] Halpern F, Horacek J, Pitts R and Ricci P 2016 Plasma Physics and Controlled Fusion $\mathbf{5 8} 084003$

[91] Olsen J, Nielsen A H, Rasmussen J J, Madsen J, Eich T, Sieglin B and Naulin V 2018 Plasma Physics and Controlled Fusion 60085018 
[92] Sieglin B, Eich T, Faitsch M, Herrmann A, Scarabosio A et al. 2016 Plasma Physics and Controlled Fusion 58055015

[93] Militello F, Naulin V and Nielsen A H 2013 Plasma Physics and Controlled Fusion 55074010

[94] Myra J, Russell D, D'Ippolito D, Ahn J W, Maingi R, Maqueda R, Lundberg D, Stotler D, Zweben S, Boedo J et al. 2011 Physics of Plasmas 18012305

[95] Fundamenski W 2005 Plasma physics and controlled fusion 47 R163

[96] Garcia L and Carreras B A 2020 Plasma Physics and Controlled Fusion 62115013

[97] Braginskii S 1965 Reviews of plasma physics $\mathbf{1}$

[98] Braginskii S 1958 Sov. Phys. JETP 6 358-369

[99] Wesson J and Campbell D J 2011 Tokamaks vol 149 (Oxford university press)

[100] Bodi K, Ciraolo G, Ghendrih P, Schwander F, Serre E and Tamain P 2011 Impact of the boussinesq approximation in tokamak scrape-off layer turbulence 38th EPS Conference on Plasma Physics $P$ vol 1 p 121

[101] Nedospasov A, Petrov V and Fidel'Man G 1985 Nuclear fusion 2521 